Accepted Manuscript

The Elemental Abundances (with Uncertainties) of the Most Earth-like Planet

Haiyang Wang, Charles H. Lineweaver, Trevor R. Ireland
PII:

DOI: S0019-1035(17)30222-1

10.1016/j.icarus.2017.08.024

Reference:

YICAR 12579

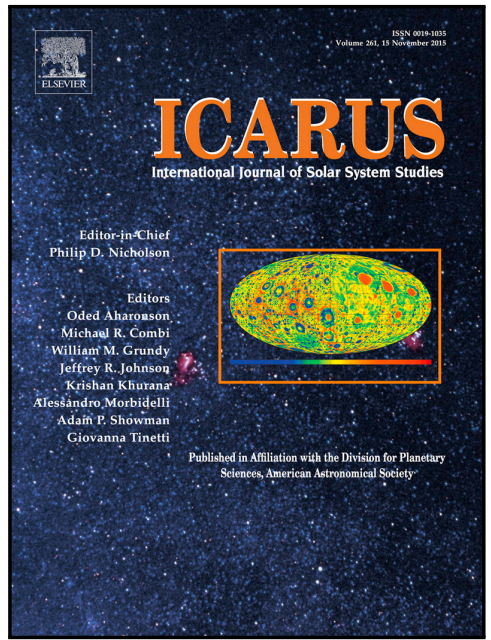

To appear in:

Icarus

Received date:

17 March 2017

Revised date:

7 August 2017

Accepted date:

Please cite this article as: Haiyang Wang, Charles H. Lineweaver, Trevor R. Ireland, The Elemental Abundances (with Uncertainties) of the Most Earth-like Planet, Icarus (2017), doi: 10.1016/j.icarus.2017.08.024

This is a PDF file of an unedited manuscript that has been accepted for publication. As a service to our customers we are providing this early version of the manuscript. The manuscript will undergo copyediting, typesetting, and review of the resulting proof before it is published in its final form. Please note that during the production process errors may be discovered which could affect the content, and all legal disclaimers that apply to the journal pertain. 


\section{Highlights}

- We present a set of concordance estimates (with uncertainties) for the elemental abundances of the primitive mantle, core, and Bulk Earth.

- The uncertainties on our elemental abundances calibrate the unresolved discrepancies between standard Earth models under various geochemical and geophysical assumptions.

- This set of concordance estimates (with uncertainties) provides a reference that can be used to compare the Earth to the Sun, which will lead to a more precise devolatilization pattern, potentially applicable to exoplanets and their host stars. 


\title{
The Elemental Abundances (with Uncertainties) of the Most Earth-like Planet
}

\author{
Haiyang Wang ${ }^{\mathrm{a}, \mathrm{b}, *}$, Charles H. Lineweaver ${ }^{\mathrm{a}, \mathrm{b}, \mathrm{c}}$, Trevor R. Ireland $\mathrm{d}^{\mathrm{b}, \mathrm{c}}$ \\ ${ }^{a}$ Research School of Astronomy and Astrophysics, The Australian National University, Canberra, ACT 2611, Australia \\ ${ }^{b}$ Planetary Science Institute, The Australian National University, Canberra, ACT 2611, Australia \\ ${ }^{c}$ Research School of Earth Sciences, The Australian National University, Canberra, ACT 2601, Australia
}

\begin{abstract}
To first order, the Earth as well as other rocky planets in the Solar System and rocky exoplanets orbiting other stars, are refractory pieces of the stellar nebula out of which they formed. To estimate the chemical composition of rocky exoplanets based on their stellar hosts' elemental abundances, we need a better understanding of the devolatilization that produced the Earth. To quantify the chemical relationships between the Earth, the Sun and other bodies in the Solar System, the elemental abundances of the bulk Earth are required. The key to comparing Earth's composition with those of other objects is to have a determination of the bulk composition with an appropriate estimate of uncertainties. Here we present concordance estimates (with uncertainties) of the elemental abundances of the bulk Earth, which can be used in such studies. First we compile, combine and renormalize a large set of heterogeneous literature values of the primitive mantle (PM) and of the core. We then integrate standard radial density profiles of the Earth and renormalize them to the current best estimate for the mass of the Earth. Using estimates of the uncertainties in i) the density profiles, ii) the core-mantle boundary and iii) the inner core boundary, we employ standard error propagation to obtain a core mass fraction of $32.5 \pm 0.3 \mathrm{wt} \%$. Our bulk Earth abundances are the weighted sum of our concordance core abundances and concordance PM abundances. Unlike previous efforts, the uncertainty on the core mass fraction is propagated to the uncertainties on the bulk Earth elemental abundances. Our concordance estimates for the abundances of $\mathrm{Mg}, \mathrm{Sn}, \mathrm{Br}, \mathrm{B}, \mathrm{Cd}$ and $\mathrm{Be}$ are significantly lower than previous estimates of the bulk Earth. Our concordance estimates for the abundances of $\mathrm{Na}, \mathrm{K}, \mathrm{Cl}, \mathrm{Zn}, \mathrm{Sr}, \mathrm{F}, \mathrm{Ga}, \mathrm{Rb}, \mathrm{Nb}, \mathrm{Gd}, \mathrm{Ta}, \mathrm{He}, \mathrm{Ar}$, and $\mathrm{Kr}$ are significantly higher. The uncertainties on our elemental abundances usefully calibrate the unresolved discrepancies between standard Earth models under various geochemical and geophysical assumptions.
\end{abstract}

Keywords: Bulk Earth, Primitive mantle, Core, Elemental composition, Exoplanet

\section{Introduction}

The number of known rocky exoplanets is rapidly increasing. Transit photometry and radial velocity measurements, when combined, yield rough estimates of the densities and therefore mineralogies of these exoplanets. Independent and potentially more precise estimates of the chemical composition of these rocky planets can be made based on the known elemental abundances of their host stars combined with estimates of the devolatilization process that produced the rocky planets from their stellar nebulae. To proceed with this strategy, we need to quantify the devolatilization that produced the Earth from the solar nebula. Knowledge of the bulk elemental abundances of the Earth with uncertainties is an important part of this research. The elemental abundances of bulk Earth (including both the bulk silicate Earth and the core) can tell us a more complete story of the potentially universal accretion and fractionation processes that produce rocky planets from nebular gas during star formation. Uncertainties associated with the bulk Earth composition are needed to compare and quantify compositional differences between the Earth, Sun, and other solar system bodies. Such comparisons can lead to a more detailed understanding of devolatilization and

${ }^{*}$ Corresponding author. Mailing address: Mt Stromlo Observatory (ANU Research School of Astronomy and Astrophysics), Cotter Road, Weston Creek, ACT 2611, Australia

Email address: haiyang . wang@anu.edu. au (Haiyang Wang) 
the chemical relationship between a terrestrial planet and its host star. The bulk Earth elemental abundances will help determine what mixture of meteorites, comets and other material produced the Earth (Drake and Righter, 2002; Burbine and O'Brien, 2004) and can also help determine the width of the feeding zone of the Earth in the protoplanetary disk (Chambers, 2001; Kaib and Cowan, 2015).

There are many stages of compositional fractionation between the collapse of a stellar nebula, the evolution of a proto-planetary disk and a final rocky planet. Composition- and position-dependent differences in the duration and strength of the various fractionating processes can lead to a variety of outcomes. The different (but somewhat similar) compositions of Earth, Mars and Vesta are a measure of these variations within our own Solar System.

A major challenge to estimating the bulk chemical composition of the Earth is that we can only sample the upper part of the possibly heterogeneous mantle, and we have no direct access to its deep interior, and even less to the core (Allègre et al., 2001). Early studies on primitive mantle (PM) elemental abundances include O'Neill (1991), Kargel and Lewis (1993), McDonough and Sun (1995), and O'Neill and Palme (1998). Bulk elemental abundances with uncertainties (Allègre et al., 2001) were reported 16 years ago but much work on PM abundances and on core abundances (usually separately) has been done since then (e.g., Lyubetskaya and Korenaga, 2007; Palme and O'Neill, 2014; Rubie et al., 2011; Wood et al., 2013; Hirose et al., 2013). The determination of uncertainties is central to the quantification of elemental abundances but has been a missing priority in previous work. The most recent, highly cited estimates of the elemental abundances of the bulk Earth do not include uncertainties (McDonough, 2003; McDonough and Arevalo, 2008).

Elemental abundance discrepancies are in large part model-dependent (McDonough, 2016), but over the past 15 years progress has been made in making more plausible models. Our knowledge of the core (and therefore of the bulk Earth) has increased significantly: high pressure experiments yield improved partition coefficients of siderophiles (Siebert et al., 2013) and improved affinities of light elements for iron (Ricolleau et al., 2011; Mookherjee et al., 2011). Seismic velocites through the core provide increasingly precise constraints on densities and on mineral physics models (Vočadlo, 2007; Li and Fei, 2014; Badro et al., 2014). Better subduction models (Poitrasson and Zambardi, 2015) and estimates of the degree of homogeneity of the mantle (Javoy et al., 2010; Nakajima and Stevenson, 2015; McDonough, 2016) provide new constraints that are being included in the upper and lower mantle abundance estimates. Better observations of geo-neutrinos (Bellini et al., 2010; Gando et a1., 2011; Bellini et al., 2013; Gando et al., 2013) provide new thermal constraints for the abundances of heat-producing elements in the Earth (e.g. Sramek et al., 2013; Huang et al., 2013). The large majority of the literature on elemental abundances of the Earth, involving either the analysis of the PM or of the core, are increasingly important and when combined, yield improved elemental abundances of the bulk Earth composition and more realistic uncertainties.

Our main research goal is to analyze the compositional differences between the Earth, Sun and other solar system bodies and from this comparison quantify the devolatilization of stellar material that leads to rocky planets. This requires estimates of the bulk Earth abundances with uncertainties. These bulk abundances and their uncertainties are poorly constrained and often ignored in the literature. Motivated by this, we make a concordance estimate of the bulk Earth elemental abundances and their uncertainties. The words "concordance estimate" specifically mean a compositional estimate that is representative of, and concordant with previous estimates. The aim of this work therefore is not to resolve the discrepancies between competing models and assumptions but to construct a concordance model (with uncertainties) that represents current knowledge of bulk Earth composition and calibrates unresolved discrepancies. We envisage that if the discrepancies can be resolved, a better formulation for estimating uncertainties might be forthcoming. However, at this stage, some of the arguments concerning the derivation of models, or values resulting from these models, appear intractable. Nevertheless, there is an essential need for uncertainties in the estimates if we are to make progress in comparing planetary objects.

We organize this paper as follows. In Sect. 2 we present the concordance estimate for the composition of PM. In Sect. 3 we present the concordance estimate of the core; in Sect. 4 we make a new estimate (with uncertainty) of the core mass fraction of the Earth, using it as the weighting factor to combine the PM with the core to yield our concordance estimates for the bulk Earth. In Sect. 5 we discuss details of how our work differs from previous work and some unresolved issues that might affect our results. 


\section{Composition of the Primitive Mantle}

\subsection{Data sources}

Earth's primitive mantle (PM) or bulk silicate Earth (BSE) is the mantle existing after core segregation but before the extraction of continental and oceanic crust and the degassing of volatiles (Sun, 1982; Kargel and Lewis, 1993; Saal et al., 2002; McDonough, 2003; Lyubetskaya and Korenaga, 2007; Palme and O’Neill, 2014). There are two major, and partially-overlapping modeling strategies for estimating the PM composition.

The peridotite model is based on the analysis of chemical data from basalts and periodotite massifs. Peridotitebasalt melting trends yield an estimate of the PM composition (e.g., Ringwood, 1979; Sun, 1982; McDonough and Sun, 1995; McDonough, 2003; Lyubetskaya and Korenaga, 2007). The peridotite model has a number of intrinsic problems, including the non-uniqueness of melting trends, large scatter in the data from mid-ocean ridge basalts (MORB) and from ocean island basalts (OIB), and the difficulty of imposing multiple cosmochemical constraints on refractory lithophile element (RLE) abundances, often resulting in model-dependent, poorly quantified uncertainties (Lyubetskaya and Korenaga, 2007).

The cosmochemical model is based on the identification of Earth with a particular class of chondritic or achondritic meteorites or their mixtures (e.g., Morgan and Anders, 1980; Javoy et al., 2010; Fitoussi et al., 2016), along with a number of assumptions on accretion and fractionation processes (Allègre et al., 2001). The cosmochemical model uses chondritic ratios of RLEs and volatility trends (e.g., Wanke and Dreibus, 1988; Palme and O'Neill, 2003, 2014). Palme and O'Neill (2003) and Palme and O'Neill (2014) present a core-mantle mass balance approach for calculating the primitive mantle composition. This approach requires an accurate determination of magnesium number $(\mathrm{Mg} \#=$ molar $\mathrm{Mg} /(\mathrm{Mg}+\mathrm{Fe}))$. A reasonable range for $\mathrm{Mg} \#$ can be inferred from fertile mantle periodites.

Our aim is not to resolve the differences between these strategies and models but to construct a concordance model whose mean values and uncertainties adequately represent current knowledge and disagreement.

\subsection{Concordance PM Estimate}

We construct our concordance PM abundances from three major papers reporting PM abundances (Lyubetskaya and Korenaga, 2007; McDonough and Arevalo, 2008; Palme and O'Neill, 2014), supplemented with noble gas abundances from Marty (2012) and Halliday (2013). McDonough and Arevalo (2008) is an updated version of their pioneering peridotite model (McDonough and Sun, 1995; McDonough, 2003). Updates of some abundances, for example W, K and Pb, can be found in Arevalo and McDonough (2008) and Arevalo et al. (2009). Lyubetskaya and Korenaga (2007) performed a principal component analysis of the same peridodite database but with different model parameters. Palme and O'Neill (2014) is largely based on a cosmochemical model using mass balance and is an updated version of their pioneering earlier work (Palme and O’Neill, 2003).

Challenges to combining these three data sets are:

i) McDonough and Areyalo (2008) report no uncertainties, however the PM abundance uncertainties reported in McDonough and Sun (1995) approximately reflect current uncertainties. Thus, in our analysis, we attach them to the PM abundances reported in McDonough and Arevalo (2008).

ii) Lyubetskaya and Korenaga (2007) do not report abundances of four volatiles; C, H, N, O.

iii) Palme and O'Neill (2014) do not report uncertainties for C, N, Se, Te, In, Hg and Bi, while Lyubetskaya and Korenaga (2007) do not report uncertainties for Te, In, Hg, Bi, Ag, Cd and Tl. We assign the McDonough and Sun (1995) uncertainties to these elements.

iv) none of these three PM models include noble gas abundances. In a preliminary attempt to be more comprehensive, we supplement these three PM models with recent noble gas abundances: the atmospheric model of Marty (2012) and three different models (layered mantle, impact erosion, and basaltic glass) from Halliday (2013). This range encompasses recent results from Dauphas and Morbidelli (2014) (Marty et al., 2016). See Appendix A.1 for details.

The resultant concordance estimates for the PM elemental abundances and their uncertainties, are listed in column 3 of Table 1. Our PM elemental abundances are increased by $0.3 \%$ to ensure that the sum of all the ppm values equals $10^{6}$ (see Appendix C). Figs. $1 \& 2$ show the comparison of our concordance abundances with the literature values. The upper panel of Fig. 1 shows elemental abundances (ppm by mass). The literature abundances normalized to our concordance PM abundances are shown in the lower panel. For clarity, Fig. 2 is a zoomed-in version of the 13 most abundant elements in Fig. 1. The abundances of these 13 elements account for $99.93_{-0.72}^{+0.07} \mathrm{wt} \%$ of primitive mantle. 
By construction, our concordance PM abundances are consistent with the literature abundances. There are some outliers. For example, the Lyubetskaya and Korenaga (2007) abundances of $\mathrm{Cl}$ and $\mathrm{Br}$ are relatively low because they used the $\mathrm{Cl} / \mathrm{K}$ ratio of $0.0075 \pm 0.0025$ from highly depleted MORB in Saal et al. (2002). This ratio is $\sim 10$ times lower than an equivalent $\mathrm{Cl} / \mathrm{K}$ ratio of $\sim 0.07$ used in McDonough and Arevalo (2008) (which came from the $\mathrm{Cl} / \mathrm{Rb} \sim 28$ and $\mathrm{K} / \mathrm{Rb} \sim 400$ of McDonough and Sun, 1995). The abundances of K estimated in Lyubetskaya and Korenaga (2007)

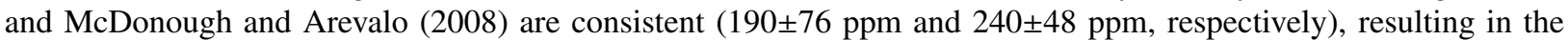
abundance of $\mathrm{Cl}$ in Lyubetskaya and Korenaga (2007) 12 times lower than that in McDonough and Arevalo (2008). Based on a 10\% partial melting MORB and the mass balance, Palme and O'Neill (2014) estimate a total CI content of 30 ppm for PM, $\sim 21$ times higher that that $(\sim 1.4 \mathrm{ppm})$ in Lyubetskaya and Korenaga (2007). The Lyubetskaya and Korenaga (2007) abundance of $\mathrm{Br}$ comes from the $\mathrm{Cl} / \mathrm{Br}$ ratio of $\sim 400 \pm 50$, which is the same ratio used in Palme and O'Neill (2014) and is higher than the approximate 350 (McDonough and Arevalo, 2008; McDonough and Sun, 1995). Thus, the Lyubetskaya and Korenaga (2007) Br abundance is also low and inconsistent with that of McDonough and Arevalo (2008) and of Palme and O'Neill (2014). Because of this inconsistency, we use an unweighted mean for Cl and $\mathrm{Br}$ (see Appendix A.1). As a result, the ratio of our estimated $\mathrm{Cl}$ and $\mathrm{Br}$ abundances is $\sim 376$.

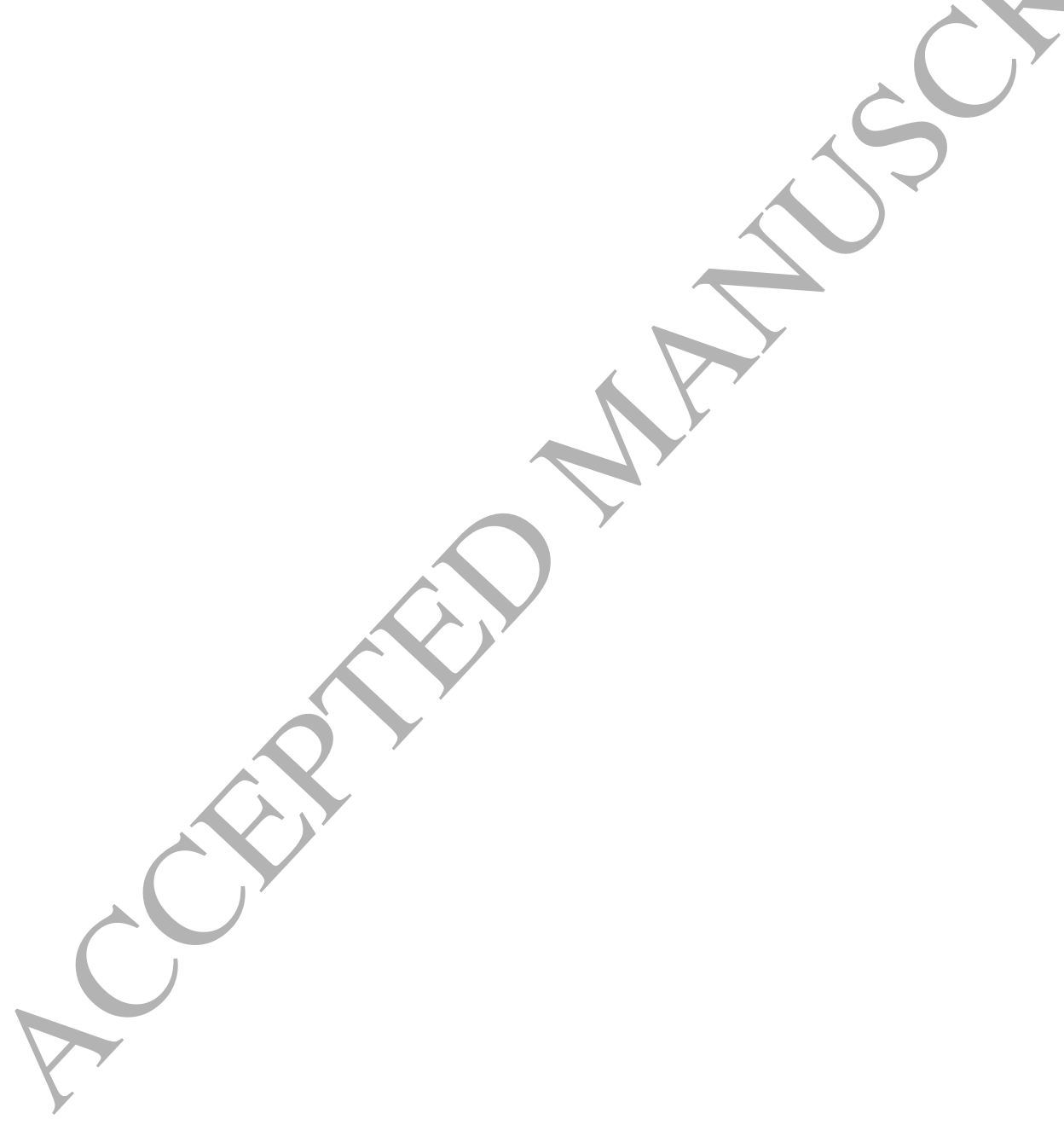



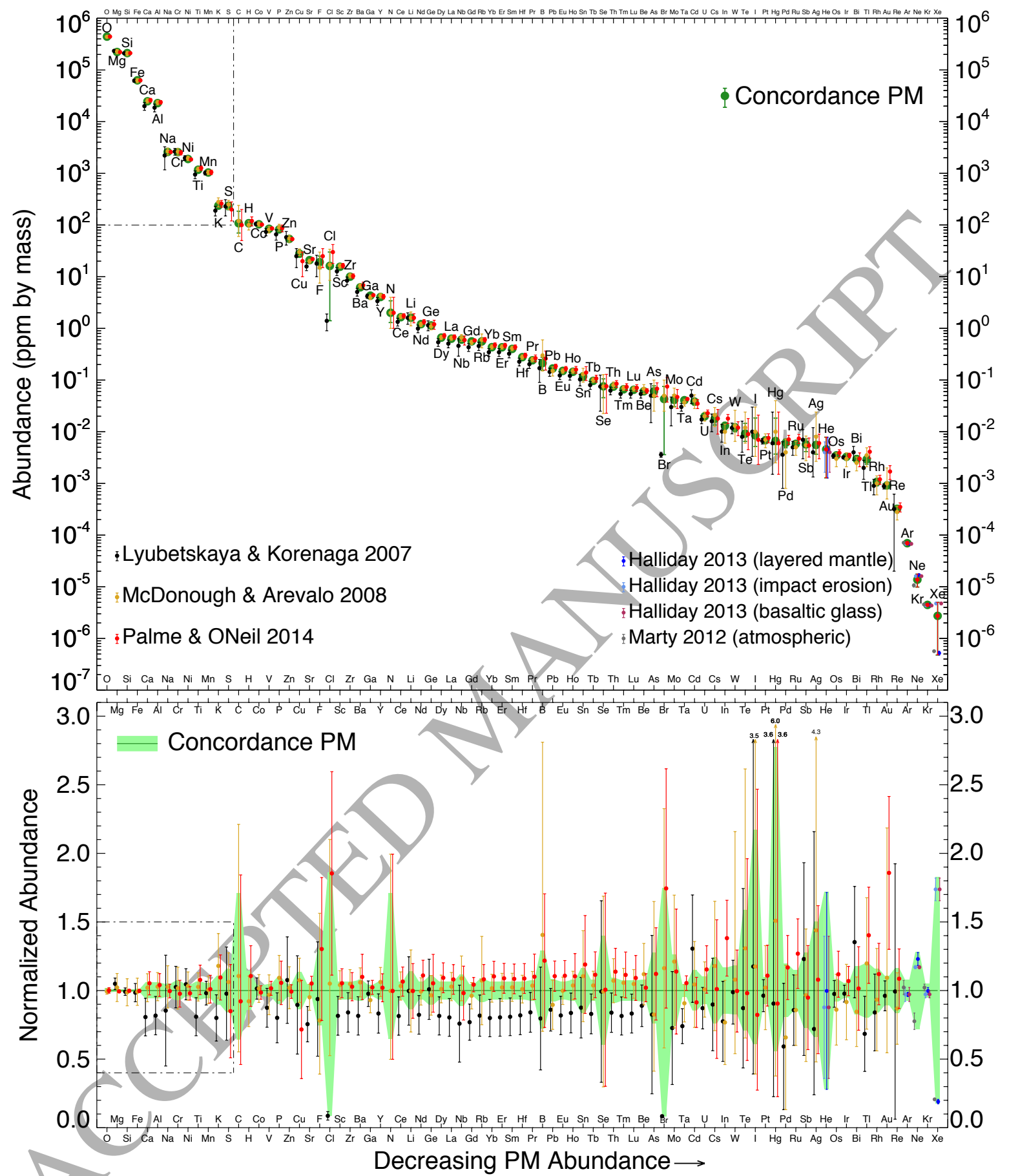

Figure 1: Recent literature estimates of 83 elemental abundances in the primitive mantle (PM) and our concordance estimates (green) constructed from them. Elements are plotted in order of decreasing PM abundance. The upper panel plots ppm by mass. In the lower panel, literature values have been normalized to our PM concordance estimates. Our PM concordance ppm estimates have been rescaled up by $0.3 \%$ to ensure that their sum equals $10^{6}$. We have not rescaled the literature values (see Appendix $\mathrm{C}$ for rescaling details). The light green band in the lower panel indicates our estimate of the uncertainties on the concordance values. $64 \%$ of the literature points fall within this band. The string of relatively low values for RLEs in the middle of the plot for the Lyubetskaya and Korenaga (2007) data set is due to its relatively high Mg abundance. Upper limits that extend beyond the plot range are labeled with their y-values. The dashed boxes on the left in both panels contain the 13 most abundant elements and are zoomed-in on in Fig. 2. 


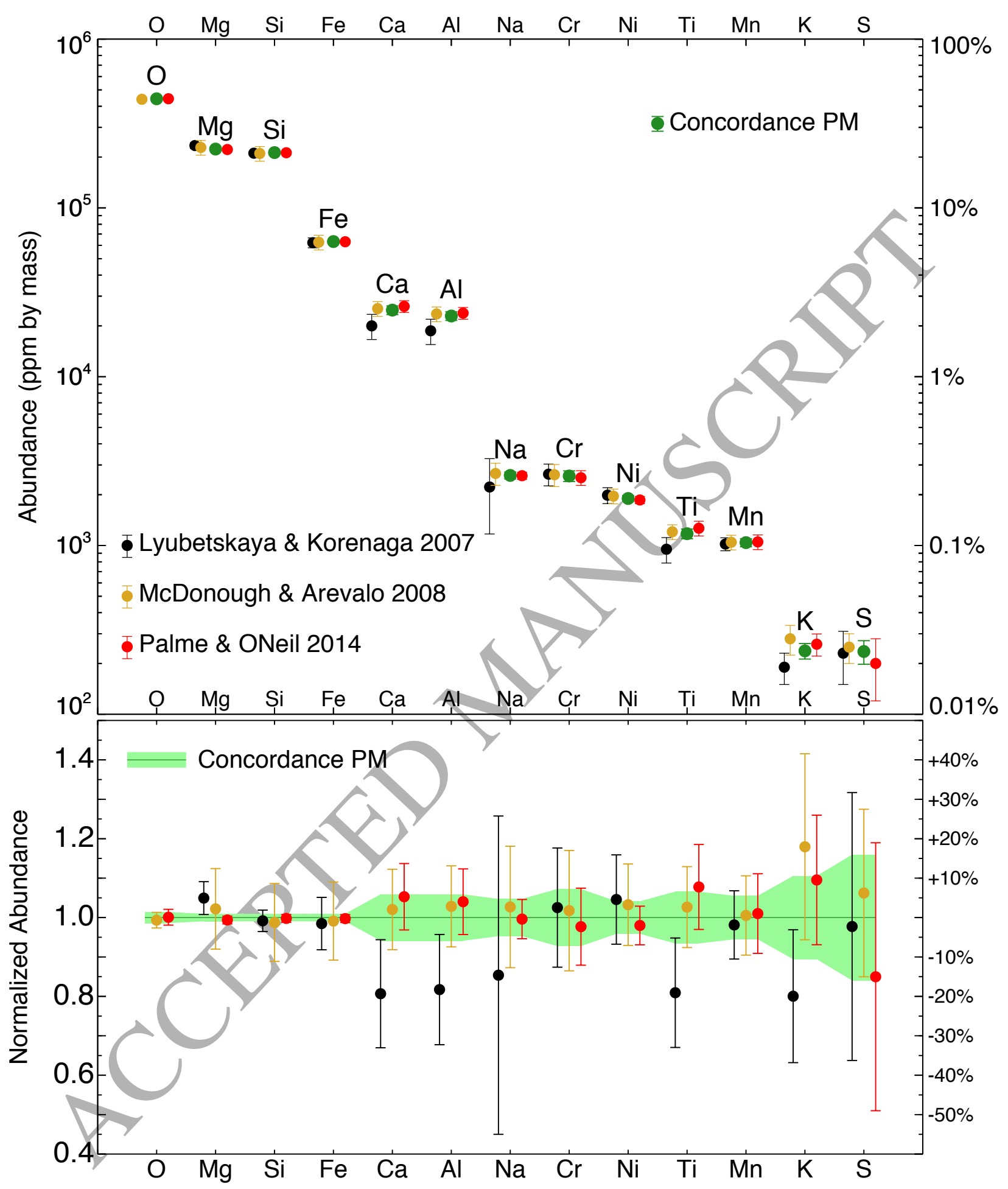

Figure 2: Zoom-in of the 13 most abundant elements contained in the dashed boxes in both panels of the previous figure. The sum of the abundances of the 4 most abundant elements $(\mathrm{O}, \mathrm{Mg}, \mathrm{Si}, \mathrm{Fe})$ make up $94.19 \pm 0.69 \%$ of the total PM mass. The 6 most abundant elements $(\mathrm{O}, \mathrm{Mg}$, $\mathrm{Si}, \mathrm{Fe}, \mathrm{Ca}$, Al) make up $98.96 \pm 0.72 \%$, while the 13 most abundant elements plotted here make up $99.93_{-0.72}^{+0.07}$ of the total PM mass. The extra mass in Mg in the Lyubetskaya and Korenaga (2007) data set is compensated for by their low values of Ca and Al. This is a good example of the covariance or non-independence of elemental abundance estimates. 
Table 1: Concordance estimates of the elemental abundances of primitive mantle, core, and bulk Earth. Following convention, depending on the element, we use ppm (by mass) or $w t \%$ or ppb (as indicated in column 2).

\begin{tabular}{|c|c|c|c|c|c|c|c|c|c|}
\hline \multirow[t]{2}{*}{ Z } & \multirow{2}{*}{$\begin{array}{l}\text { Elem } \\
\mathrm{H}\end{array}$} & $\begin{array}{l}\text { Concordance Prim } \\
\text { abundance (ppm) }{ }^{a}\end{array}$ & nitive Mantle & Sources $^{b}$ & $\begin{array}{l}\text { Concordance Core } \\
\text { abundance (ppm) }{ }^{a}\end{array}$ & & Sources $^{c}$ & $\begin{array}{l}\text { Bulk Earth } \\
\text { abundance (pp }\end{array}$ & $\mathrm{pm}^{a}$ \\
\hline & & 109 & \pm 15 & MA08, PO14 & 569 & \pm 421 & W06, MA08, ZY12 & 258 & \pm 137 \\
\hline 2 & $\mathrm{He}(\mathrm{ppb})$ & 4.56 & \pm 3.29 & $\mathrm{H} 13$ & . & & - & 3.08 & \pm 2.22 \\
\hline 3 & $\mathrm{Li}$ & 1.60 & \pm 0.22 & LK07, MA08, PO14 & 1.57 & \pm 0.59 & KL93 & 1.59 & \pm 0.24 \\
\hline 4 & $\mathrm{Be}$ & 0.0607 & \pm 0.0048 & LK07 & - & & - & 0.0410 & \pm 0.0033 \\
\hline 5 & B & 0.213 & ${ }_{-0.059}^{+0.062}$ & LK07, MA08, PO14 & - & & - & 0.144 & ${ }_{-0.040}^{+0.042}$ \\
\hline 6 & $\mathrm{C}$ & 109 & $\begin{array}{ll}7770 \\
-39\end{array}$ & MA08, PO14 & 7921 & \pm 6820 & W06, MA08, ZY12, W13, N15, LS16 & 2648 & \pm 2217 \\
\hline 7 & $\mathrm{~N}$ & 2.01 & $\begin{array}{l}-3.42 \\
+1.42\end{array}$ & MA08, PO14 & 93 & +77 & MA08, ZY12 & 31.7 & $\begin{array}{r}+24.9 \\
-203\end{array}$ \\
\hline 8 & $\mathrm{O}(\%)$ & 44.3 & $\begin{array}{l}-0.71 \\
\pm 0.6\end{array}$ & MA08, PO14 & 2.66 & \pm 1.82 & KL93, A01, M03, J10, H11, R11, ZY12, H13, S13, B14, LS16 & 30.8 & $\pm 0.7^{-3}$ \\
\hline 9 & $\mathrm{~F}$ & 19.2 & ${ }_{-4.9}^{+5.8}$ & LK07, MA08, PO14 & - & & - & 13.0 & +3.9 \\
\hline 10 & $\mathrm{Ne}(\mathrm{ppb})$ & 0.0137 & \pm 0.0039 & M12, H13 & - & & - & 0.0092 & $27 \pm 0.0026$ \\
\hline 11 & $\mathrm{Na}$ & 2600 & \pm 123 & LK07, MA08, PO14 & 1372 & \pm 519 & KL93 & 2201 & \pm 188 \\
\hline 12 & $\operatorname{Mg}(\%)$ & 22.3 & \pm 0.2 & LK07, MA08, PO14 & 0.0588 & ${ }_{-0.0288}^{+0.0294}$ & $\mathrm{ZY} 12$ & 15.1 & \pm 0.2 \\
\hline 13 & $\mathrm{Al}(\%)$ & 2.29 & \pm 0.13 & LK07, MA08, PO14 & - & & - $\quad-2$ & 1.54 & \pm 0.09 \\
\hline 14 & $\mathrm{Si}(\%)$ & 21.3 & \pm 0.2 & LK07, MA08, PO14 & 4.96 & \pm 2.34 & A01, W06, MA08, J10, R11, ZY12, H13, S & 16.0 & \pm 0.8 \\
\hline 15 & $\mathrm{P}$ & 82.4 & \pm 8.0 & LK07, MA08, PO14 & 2774 & $+{ }_{-1236}^{+916}$ & A01, MA08, KL93, ZY12 & 957 & ${ }_{-402}^{+298}$ \\
\hline 16 & $\mathrm{~s}$ & 235 & \pm 38 & LK07, MA08, PO14 & 18269 & $\begin{array}{l}-1236 \\
\pm 4497\end{array}$ & KL93, A01, W06, MA08, R11, H & 6096 & $\begin{array}{l}-422 \\
\pm 1463\end{array}$ \\
\hline 17 & $\mathrm{Cl}$ & 16.2 & $\begin{array}{l}+13.9 \\
-14.8\end{array}$ & LK07, MA08, PO14 & 459 & ${ }_{-259}^{+278}$ & KL93, MA08 & 160 & ${ }_{-85}^{+91}$ \\
\hline 18 & $\mathrm{Ar}(\mathrm{ppb})$ & 0.0697 & $\begin{array}{l}-14.8 \\
\pm 0.0054\end{array}$ & M12, H13 & & & - & 0.0471 & $\begin{array}{l}-85 \\
\pm 0.0036\end{array}$ \\
\hline 19 & $\mathrm{~K}$ & 237 & \pm 25 & LK07, MA08, PO14 & 206 & \pm 78 & KL93 & 227 & \pm 30 \\
\hline 20 & $\mathrm{Ca}(\%)$ & 2.48 & \pm 0.15 & LK07, MA08, PO14 & - & & - & 1.67 & \pm 0.10 \\
\hline 21 & $\mathrm{Sc}$ & 15.6 & \pm 1.0 & LK07, MA08, PO14 & - & & - & 10.5 & \pm 0.7 \\
\hline 22 & $\mathrm{Ti}$ & 1174 & \pm 77 & LK07, MA08, PO14 & - & & 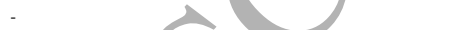 & 792 & \pm 52 \\
\hline 23 & $\mathrm{v}$ & 84.6 & \pm 3.9 & LK07, MA08, PO14 & 122 & \pm 46 & KL93, MA08, R11 & 96.6 & \pm 15.2 \\
\hline 24 & $\mathrm{Cr}$ & 2580 & \pm 187 & LK07, MA08, PO14 & 6590 & \pm 1751 & KL93, A01, MA08, J10, R11 & 3883 & \pm 583 \\
\hline 25 & $\mathrm{Mn}$ & 1040 & \pm 57 & LK07, MA08, PO14 & 3257 & +2563 & KL93, A01, M03, MA08 & 1760 & ${ }_{-944}^{+834}$ \\
\hline 26 & $\mathrm{Fe}(\%)$ & 6.32 & \pm 0.06 & LK07, MA08, PO14 & 82.8 & $\begin{array}{l}-2903 \\
\pm 2.9\end{array}$ & KL93, A01,M03, MA08, J10, R1I & 31.2 & $\begin{array}{l}-944 \\
\pm 1.0\end{array}$ \\
\hline 27 & Co & 103 & \pm 4 & LK07, MA08, PO14 & 2373 & \pm 141 & KL93, A01, MA08, J10, R11 & 841 & \pm 46 \\
\hline 28 & $\mathrm{Ni}(\%)$ & 0.190 & \pm 0.008 & LK07, MA08, PO14 & 5.06 & \pm 0.23 & $\mathrm{KL} 93, \mathrm{~A} 01, \mathrm{M} 03, \mathrm{MA08}, \mathrm{J} 10, \mathrm{R} 11$ & 1.77 & \pm 0.08 \\
\hline 29 & $\mathrm{Cu}$ & 27.9 & \pm 3.8 & LK07, MA08, PO14 & 154 & \pm 58 & MA08, KL93 & 69.0 & \pm 19.2 \\
\hline 30 & $\mathrm{Zn}$ & 53.9 & \pm 2.5 & LK07, MA08, PO14 & 29 & \pm 11 & KL93 & 45.9 & \pm 4.0 \\
\hline 31 & $\mathrm{Ga}$ & 4.30 & \pm 0.17 & LK07, MA08, PO14 & 4.90 & \pm 1.85 & KL93 & 4.49 & \pm 0.61 \\
\hline 32 & $\mathrm{Ge}$ & 1.14 & \pm 0.12 & LK07, MA08, PO14 & 24.0 & \pm 9.1 & KL93, MA08 & 8.56 & \pm 2.95 \\
\hline 33 & As & 0.0606 & +0.0167 & LK07, MA08, PO14 & 4.89 & \pm 1.85 & KL93, MA08 & 1.63 & \pm 0.60 \\
\hline 34 & $\mathrm{Se}$ & 0.0755 & $\begin{array}{l}-0.0145 \\
\pm 0.0300\end{array}$ & LK07, MA08, PO14 & 8.63 & \pm 3.27 & KL93, MA08 & 2.86 & \pm 1.06 \\
\hline 35 & $\mathrm{Br}$ & 0.0430 & ${ }_{-0.0394}^{+0.0322}$ & LK07, MA08, PO14 & 0.514 & \pm 0.195 & KL93, MA08 & 0.196 & ${ }_{-0.069}^{+0.067}$ \\
\hline 36 & $\mathrm{Kr}(\mathrm{ppb})$ & 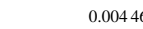 & $16 \pm 0.00022$ & M12, H13 & & & & 0.0030 & $\begin{array}{r}-0.069 \\
01 \pm 0.0001\end{array}$ \\
\hline 37 & $\mathrm{Rb}$ & 0.559 & \pm 0.048 & LK07, MA08, PO14 & 1.08 & \pm 0.4 & KL93 & 0.728 & \pm 0.136 \\
\hline 38 & $\mathrm{Sr}$ & 20.9 & \pm 0.9 & LK07, MA08, PO14 & & & - & 14.1 & \pm 0.6 \\
\hline 39 & $\mathrm{Y}$ & 4.05 & \pm 0.27 & LK07, MA08, PO14 & & & - & 2.73 & \pm 0.18 \\
\hline 40 & $\mathrm{Zr}$ & 10.02 & \pm 0.66 & LK07, MA08, PO14 & & & - & 6.76 & \pm 0.44 \\
\hline 41 & $\mathrm{Nb}$ & 0.606 & \pm 0.070 & LK07, MA08, PO14 & 0.423 & \pm 0.160 & R11 & 0.547 & \pm 0.070 \\
\hline 42 & Mo & 0.0413 & \pm 0.0107 & LK07, MA08, PO14 & 4.97 & \pm 1.88 & KL93, MA08 & 1.64 & \pm 0.61 \\
\hline 44 & $\mathrm{Ru}(\mathrm{ppb})$ & 5.83 & \pm 0.86 & LK07, MA08, PO14 & 3939 & $\begin{array}{l} \pm 1491 \\
\end{array}$ & KL93, MA08 & 1284 & \pm 485 \\
\hline 45 & $\mathrm{Rh}(\mathrm{ppb})$ & 1.07 & \pm 0.17 & LK07, MA08, PO14 & 684 & $\begin{array}{l} \pm 259 \\
\pm 259\end{array}$ & KL93, MA08 & 223 & $\begin{array}{l}+463 \\
\pm 84\end{array}$ \\
\hline 46 & $\mathrm{Pd}(\mathrm{ppb})$ & 6.08 & \pm 1.18 & LK07, MA08, PO14 & 2719 & \pm 1029 & KL93, MA08 & 888 & \pm 335 \\
\hline 47 & $\mathrm{Ag}(\mathrm{ppb})$ & 5.56 & $\begin{array}{l}+2.79 \\
-1.89\end{array}$ & LK07, MA08, PO14 & 239 & \pm 90 & KL93, MA08 & 81.3 & \pm 29.4 \\
\hline 48 & $\mathrm{Cd}$ & 0.0383 & $\begin{array}{l}-1.89 \\
\pm 0.0056\end{array}$ & LK07, MA08, PO14 & 0.167 & \pm 0.063 & KL93, MA08 & 0.0800 & \pm 0.0208 \\
\hline 49 & In & 0.0130 & \pm 0.0022 & LK07, MA08, PO14 & . & & & 0.0087 & $79 \pm 0.0014$ \\
\hline 50 & $\mathrm{Sn}$ & 0.118 & \pm 0.019 & LK07, MA08, PO14 & 0.490 & \pm 0.185 & KL93, MA08 & 0.239 & \pm 0.062 \\
\hline 51 & $\mathrm{Sb}(\mathrm{ppb})$ & 5.69 & \pm 1.57 & LK07, MA08, PO14 & 132 & \pm 50 & KL93, MA08 & 46.8 & \pm 16.3 \\
\hline 52 & $\mathrm{Te}(\mathrm{ppb})$ & 9.18 & $\begin{array}{l}+5.37 \\
-2.68\end{array}$ & EK07, MA08, PO14 & 1009 & \pm 382 & KL93, MA08 & 334 & \pm 124 \\
\hline 53 & $\mathrm{I}(\mathrm{ppb})$ & 8.51 & $\begin{array}{l}+9.98 \\
+9.93\end{array}$ & LK07, MA08, PO14 & 92.1 & \pm 34.9 & KL93, MA08 & 35.7 & +13.2 \\
\hline 54 & $\mathrm{Xe}(\mathrm{ppb})$ & 0.0027 & $\begin{array}{l}-3.33 \\
4 \pm 0.00226\end{array}$ & $\mathrm{M} 12, \mathrm{H} 13$ & - & & - & 0.0018 & $\begin{array}{l}-1.6 \\
85 \pm 0.0015\end{array}$ \\
\hline 55 & Cs & 0.0178 & \pm 0.0043 & LK07, MA08, PO14 & 0.103 & \pm 0.039 & KL93, MA08 & 0.0455 & \pm 0.0130 \\
\hline 56 & $\mathrm{Ba}$ & 6.23 & \pm 0.47 & LK07, MA08, PO14 & - & & - & 4.21 & \pm 0.32 \\
\hline 57 & $\mathrm{La}$ & 0.632 & \pm 0.042 & LK07, MA08, PO14 & - & & - & 0.427 & \pm 0.028 \\
\hline 58 & $\mathrm{Ce}$ & 1.65 & \pm 0.11 & LK07, MA08, PO14 & - & & - & 1.11 & \pm 0.07 \\
\hline 59 & $\mathrm{Pr}$ & 0.241 & \pm 0.018 & LK07, MA08, PO14 & - & & - & 0.163 & \pm 0.012 \\
\hline 60 & $\mathrm{Nd}$ & 1.21 & $\pm 0,08$ & LK07, MA08, PO14 & - & & - & 0.815 & \pm 0.054 \\
\hline 62 & $\mathrm{Sm}$ & 0.401 & \pm 0.026 & LK07, MA08, PO14 & - & & - & 0.270 & \pm 0.018 \\
\hline 63 & $\mathrm{Eu}$ & 0.150 & \pm 0.010 & LK07, MA08, PO14 & - & & - & 0.101 & $\begin{array}{l} \pm 0.007 \\
\pm 0.007\end{array}$ \\
\hline 64 & $\mathrm{Gd}$ & 0.561 & \pm 0.024 & LK07, MA08, PO14 & - & & - & 0.379 & \pm 0.017 \\
\hline 65 & $\mathrm{~Tb}$ & 0.0964 & \pm 0.0073 & LK07, MA08, PO14 & - & & - & 0.0651 & \pm 0.0049 \\
\hline 66 & Dy & 0.662 & \pm 0.043 & LK07, MA08, PO14 & - & & - & 0.447 & \pm 0.029 \\
\hline 67 & Ho & 0.145 & \pm 0.011 & LK07, MA08, PO14 & - & & - & 0.0976 & \pm 0.0074 \\
\hline 68 & $\mathrm{Er}$ & 0.430 & \pm 0.028 & LK07, MA08, PO14 & - & & - & 0.290 & \pm 0.019 \\
\hline 69 & $\mathrm{Tm}$ & 0.0663 & \pm 0.0050 & LK07, MA08, PO14 & - & & - & 0.0447 & \pm 0.0034 \\
\hline 70 & $\mathrm{Yb}$ & 0.433 & \pm 0.028 & LK07, MA08, PO14 & - & & - & 0.292 & \pm 0.019 \\
\hline 71 & $\mathrm{Lu}$ & 0.0649 & \pm 0.0049 & LK07, MA08, PO14 & - & & - & 0.0438 & \pm 0.0033 \\
\hline 72 & $\mathrm{Hf}$ & 0.277 & \pm 0.018 & LK07, MA08, PO14 & - & & - & 0.187 & \pm 0.012 \\
\hline 73 & $\mathrm{Ta}$ & 0.0408 & \pm 0.0019 & LK07, MA08, PO14 & 0.00799 & $9_{-0.00399}^{+0.00407}$ & R11 & 0.0301 & \pm 0.0018 \\
\hline 74 & w & 0.0120 & ${ }_{-0.0022}^{+0.0022}$ & LK07, MA08, PO14 & 0.502 & \pm 0.190 & KL93, MA08, R11 & 0.171 & \pm 0.062 \\
\hline 75 & $\operatorname{Re}(\mathrm{ppb})$ & 0.322 & $\begin{array}{l}-0.0021 \\
\pm 0.053\end{array}$ & LK07, MA08, PO14 & 214 & \pm 81 & KL93, MA08 & 69.8 & \pm 26.4 \\
\hline 76 & Os (ppb) & 3.49 & \pm 0.28 & LK07, MA08, PO14 & 2719 & \pm 1029 & KL93, MA08 & 886 & \pm 335 \\
\hline 77 & Ir (ppb) & 3.27 & \pm 0.17 & LK07, MA08, PO14 & 2611 & \pm 989 & KL93, MA08 & 851 & \pm 321 \\
\hline 78 & $\mathrm{Pt}(\mathrm{ppb})$ & 6.86 & \pm 0.67 & LK07, MA08, PO14 & 5448 & \pm 2063 & KL93, MA08 & 1775 & \pm 671 \\
\hline 79 & $\mathrm{Au}(\mathrm{ppb})$ & 0.915 & \pm 0.098 & LK07, MA08, PO14 & 481 & \pm 182 & KL93, MA08 & 157 & \pm 59 \\
\hline 80 & $\mathrm{Hg}(\mathrm{ppb})$ & 6.63 & ${ }_{-2.94}^{+11.75}$ & LK07, MA08, PO14 & 32.3 & $\begin{array}{l}+17.7 \\
-1663\end{array}$ & KL93, MA08 & 15.0 & $\begin{array}{l}+9.8 \\
-57\end{array}$ \\
\hline 81 & $\mathrm{Tl}(\mathrm{ppb})$ & 2.92 & $\begin{array}{l}-2.94 \\
\pm 0.58\end{array}$ & LK07, MA08, PO14 & 29.4 & $\begin{array}{l}-16.3 \\
\pm 11.1\end{array}$ & MA08 & 11.5 & $\begin{array}{l}-5.1 \\
\pm 3.6 \\
\end{array}$ \\
\hline 82 & $\mathrm{~Pb}$ & 0.167 & \pm 0.014 & LK07, MA08, PO14 & 1.715 & $\begin{array}{l}+1.585 \\
+1515\end{array}$ & KL93, MA08 & 0.670 & +0.515 \\
\hline 83 & $\mathrm{Bi}(\mathrm{ppb})$ & 2.96 & \pm 0.52 & LK07, MA08, PO14 & 19.1 & $\begin{array}{l}-110.9 \\
+109\end{array}$ & KL93, MA08 & 8.21 & $\begin{array}{l}-0.592 \\
+3.56^{2}\end{array}$ \\
\hline 90 & Th & 0.0746 & \pm 0.0068 & LK07, MA08, PO14 & - & & - & 0.0504 & $\begin{array}{l}-3.30 \\
\pm 0.0046\end{array}$ \\
\hline 92 & $\mathrm{U}$ & 0.0198 & \pm 0.0020 & LK07, MA08, PO14 & - & & - & 0.0134 & \pm 0.0013 \\
\hline & Total & 1.00 & $\times 10^{6}$ & & 1.00 & $\times 10^{6}$ & & 1.00 & $\times 10^{6}$ \\
\hline
\end{tabular}

${ }^{a}$ ppm (by mass) unless otherwise indicated in column 2. Values have been rescaled $\Sigma_{\mathrm{ppm}}=10^{6}$, see Table C.5.

${ }^{b}$ LK07: Lyubetskaya and Korenaga (2007) columns 4 and 5 of their Table 3; MA08: McDonough and Arevalo (2008) columns 2 and 6 of their Table 1; PO14: Palme and O'Neill (2014) columns 5 and 6 of their Table 4; H13: Halliday (2013) columns 7, 10 and 13 of their Table 2; M12: Marty (2012) last column of his Table 1. See Sect. 2.2 for the details of our concordance PM abundances. 6e to the literature acronyms for core abundances, see footnote a of Table 2 .

$d$ Weighted sum (Eqs. A.5 \& A.6) of concordance estimates of the abundances of primitive mantle and core. 
Table 2: Concentrations (wt $\%$ ) of the 13 most abundant elements in the core

\begin{tabular}{|c|c|c|c|c|c|c|c|c|c|c|c|c|c|}
\hline Sources $^{a}$ & $\mathrm{Fe}$ & $\mathrm{Ni}$ & $\mathrm{Si}$ & $\mathrm{O}$ & s & C & $\mathrm{Cr}$ & $\mathrm{P}$ & $\mathrm{Mn}$ & Co & $\mathrm{Na}$ & $\mathrm{Mg}$ & $\mathrm{H}$ \\
\hline KL93 & 85.55 & 4.88 & - & 5.18 & 2.69 & - & 0.45 & 0.41 & 0.347 & 0.218 & 0.14 & - & - \\
\hline A01/95 & $79.4 \pm 2.0$ & $4.87 \pm 0.30$ & 7.35 & $5.0 \pm 0.5$ & $1.21 \pm 0.20$ & - & 0.78 & 0.582 & 0.369 & 0.253 & - & - & - \\
\hline M03 & 88.3 & 5.4 & nil & 3 & - & - & - & 0.03 & - & - & - & - & - \\
\hline W06 & - & - & $4.5 \pm 0.5$ & - & 1.9 & 0.2 & - & - & - & - & - & - & 0.1 \\
\hline MA08 & 85 & 5.2 & 6.4 & nil & 1.9 & 0.2 & 0.9 & 0.005 & 0.32 & 0.25 & - & - & 0.06 \\
\hline $\mathrm{J} 10$ & $85.5 \pm 1.1$ & $5.35 \pm 0.81$ & $6.64 \pm 0.51$ & $1.99 \pm 0.46$ & - & - & $0.55 \pm 0.05$ & - & - & $0.25 \pm 0.03$ & - & - & - \\
\hline H11 & - & - & - & 0.5 & - & - & - & - & - & - & - & - & - \\
\hline R11 & $83.45 \pm 0.35$ & $5.3 \pm 0.1$ & $8.4 \pm 0.2$ & $0.655 \pm 0.185$ & 2 & - & $0.68 \pm 0.12$ & - & - & $0.24 \pm 0.01$ & - & - & - \\
\hline ZY12 & - & - & $2.0 \pm 0.2$ & $1.0 \pm 0.1$ & - & $0.4 \pm 0.3$ & - & . & $0.096 \pm 0.060$ & - & - & $0.06 \pm 0.03$ & $0.014 \pm 0.008$ \\
\hline H13 & - & - & 6 & 3 & $1.5 \pm 0.5$ & & - & - & - & - & & & - \\
\hline W13 & - & - & - & - & - & 1 & - & - & - & - & - & & - \\
\hline $\mathrm{S} 13$ & . & - & $1.85 \pm 0.35$ & $5.0 \pm 0.5$ & - & - & - & - & - & - & & . & . \\
\hline B14 & - & - & $1.94 \pm 0.21$ & $3.8 \pm 0.7$ & nil & nil & - & - & - & - & & & - \\
\hline N15 & - & - & - & & - & $1.05 \pm 0.15$ & - & - & - & - & & & - \\
\hline LS16 & . & - & $5.5 \pm 0.5$ & $0.75 \pm 0.25$ & $1.85 \pm 0.05$ & 2 & - & - & - & - & & -2 & . \\
\hline Mean $^{b}$ & $82.8 \pm 2.9$ & $5.06 \pm 0.23$ & $4.96 \pm 2.34$ & $2.66 \pm 1.82$ & $1.83 \pm 0.45$ & $0.79 \pm 0.68$ & $0.66 \pm 0.18$ & $\mathbf{0 . 3 2 6}_{-0.290}^{+0.256}$ & $\mathbf{0 . 2 7 7 _ { - 0 . 1 2 4 } ^ { + 0 . 0 9 2 }}$ & $0.237 \pm 0.014$ & & $0.06 \pm 0.03$ & $0.057 \pm 0.042$ \\
\hline
\end{tabular}

${ }^{a}$ K93: Kargel and Lewis (1993): column 6 of Table II

95/A01: Allègre et al. (1995) Table 2 updated with Allègre et al. (2001) for $\mathrm{S}$ and $\mathrm{O}$ to $1.21 \mathrm{wt} \%$ and $5 \mathrm{wt} \%$ respectively.

$\mathrm{Ni}$ in O-bearing calculations listed in Table 7.

Conclusions, $4-5 \mathrm{wt} \% \mathrm{Si}, 1.9 \mathrm{wt} \% \mathrm{~S}, 0.1 \mathrm{wt} \% \mathrm{H}$ and $0.2 \mathrm{wt} \% \mathrm{C}$

MA08: McDong and Arevalo (2008) columns 3 and 7 of Table 1

J10: Javoy et al. (2010) column 4 of Table 6

H11: Huang et al. (2011): the optimal value of $0.5 \mathrm{wt} \%$ oxygen in the liquid outer core

ZY12: Zhang and Yin (2012): abundances of O, Mg, and Si are from the M52 simulation results for the core listed in Table 2; abundances of H, C, P, and N are the average of the upper and lower limits of their models A and B H13: Hirose et al. (2013): 'preferred value' listed in Table

W13: Wood et al. (2013): Conclusions, of $\sim 1 \mathrm{wt} \%$ of carbon in the core

S13: Siebert et al. (2013): conclusion of an oxygen-rich core with 4.5 to $5.5 \mathrm{wt} \% \mathrm{O}$ and 1.5 to $2.2 \mathrm{wt} \% \mathrm{~S}$

B14: Badro et al. (2014): the best numerical fit indicated in Fig.2

N15: Nakajima et al. (2015): conclusion of 0.9-1.2 wt\% carbon in the core to match sound velocity

列

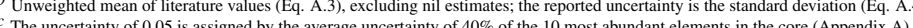

\section{Composition of the Core}

\subsection{Data sources}

The density deficit of the Earth's core (compared to a pure Fe-Ni composition) suggests that the core contains a significant amount of one or more light elements. The liquid outer core is thought to have a density deficit of $3-12$ wt\% (e.g., Stevenson, 1981; Anderson and Ahrens, 1994; Anderson and Isaak, 2002; McDonough, 2003), while the solid inner core is 3-6 wt\% less dense than predicted (e.g., Anderson and Ahrens, 1994; Hemley and Mao, 2001). The candidate light elements are still controversial and have been modeled and estimated in various ways and plausibly include Si, O, S and C (see reviews by Hirose et al., 2013; Litasov and Shatskiy, 2016).

We have compiled and combined a wide variety of recent work to construct concordance core abundances. These are listed in Tables 1 and 2 and include various constraints from various core compositional models, terrestrial fractionation curves and mass balance (e.g. Kargel and Lewis, 1993; Allègre et al., 1995; McDonough, 2003; Wood et al., 2006; McDonough and Arevalo, 2008), metal-silicate equilibrium (e.g. Rubie et al., 2011), the chemistry of core formation (e.g. Javoy et al., 2010), high-pressure and high-temperature experiments (Siebert et al., 2013), experiments based on sound veloeity and/or density jumps (Huang et al., 2011; Nakajima et al., 2015), and numerical simulations (e.g. Zhang and Yin, 2012; Badro et al., 2014).

\subsection{Concordance Core Estimate}

Since many core abundances reported in the literature are model-dependent and are given without uncertainties, our concordance abundances (Tables 1 and 2) are unweighted averages (Eq. A.3) and exclude abundances that have been set to zero. Based on mass balance between the core and the silicate Earth, McDonough (2003) proposed both Si-bearing and O-bearing core models while McDonough and Arevalo (2008) presented a Si-bearing model only. The trace elements in the McDonough (2003) O-bearing model and McDonough and Arevalo (2008) Si-bearing model are highly correlated. Therefore we only count them once in our calculations. The details of how the literature values were combined into our concordance values with uncertainties are described in Appendix A.2.

Fig. 3 shows our concordance abundances for 49 elements in the core, compared with the literature values from which they were constructed. The sum of our concordance abundances of the 49 elements is scaled to $10^{6}$ and listed in column 5 of Table 1. This is the most complete compilation for the core's composition to date. Table 2 lists the mean concordance concentrations of the 13 most abundant elements in the core along with the literature values from 
which they were constructed. Fig. 4 is a zoom-in of the 13 most abundant elements and shows this comparison in more detail. Fe-Ni alloy accounts for $87.90 \pm 2.92 \mathrm{wt} \%$ of the total mass of the core. The most abundant light element in the core is $\mathrm{Si}$, followed by $\mathrm{O}, \mathrm{S}$, and $\mathrm{C}$. The other less abundant elements include $\mathrm{Cr}, \mathrm{P}, \mathrm{Mn}, \mathrm{Co}, \mathrm{Na}, \mathrm{Mg}$ and $\mathrm{H}$.

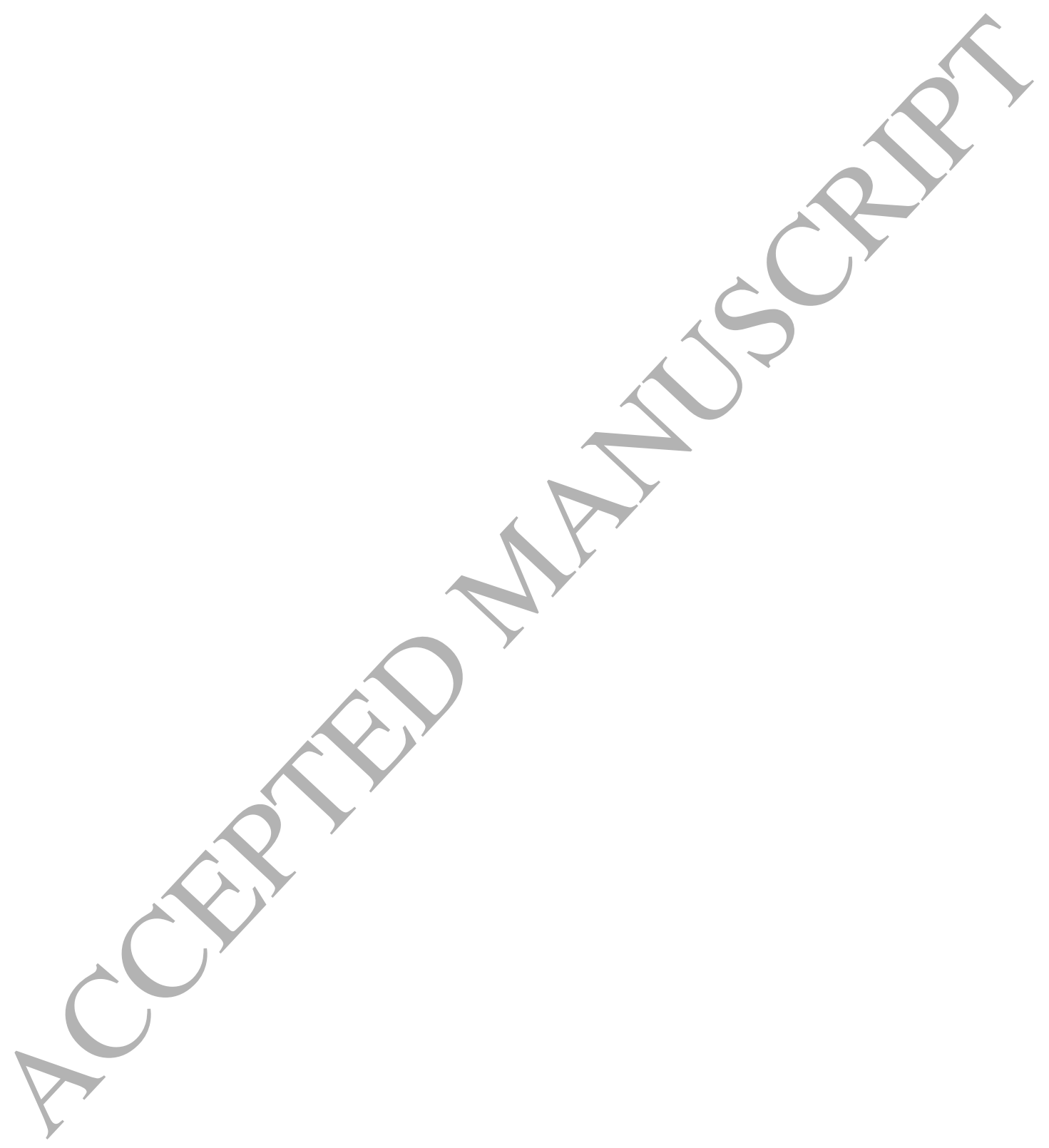




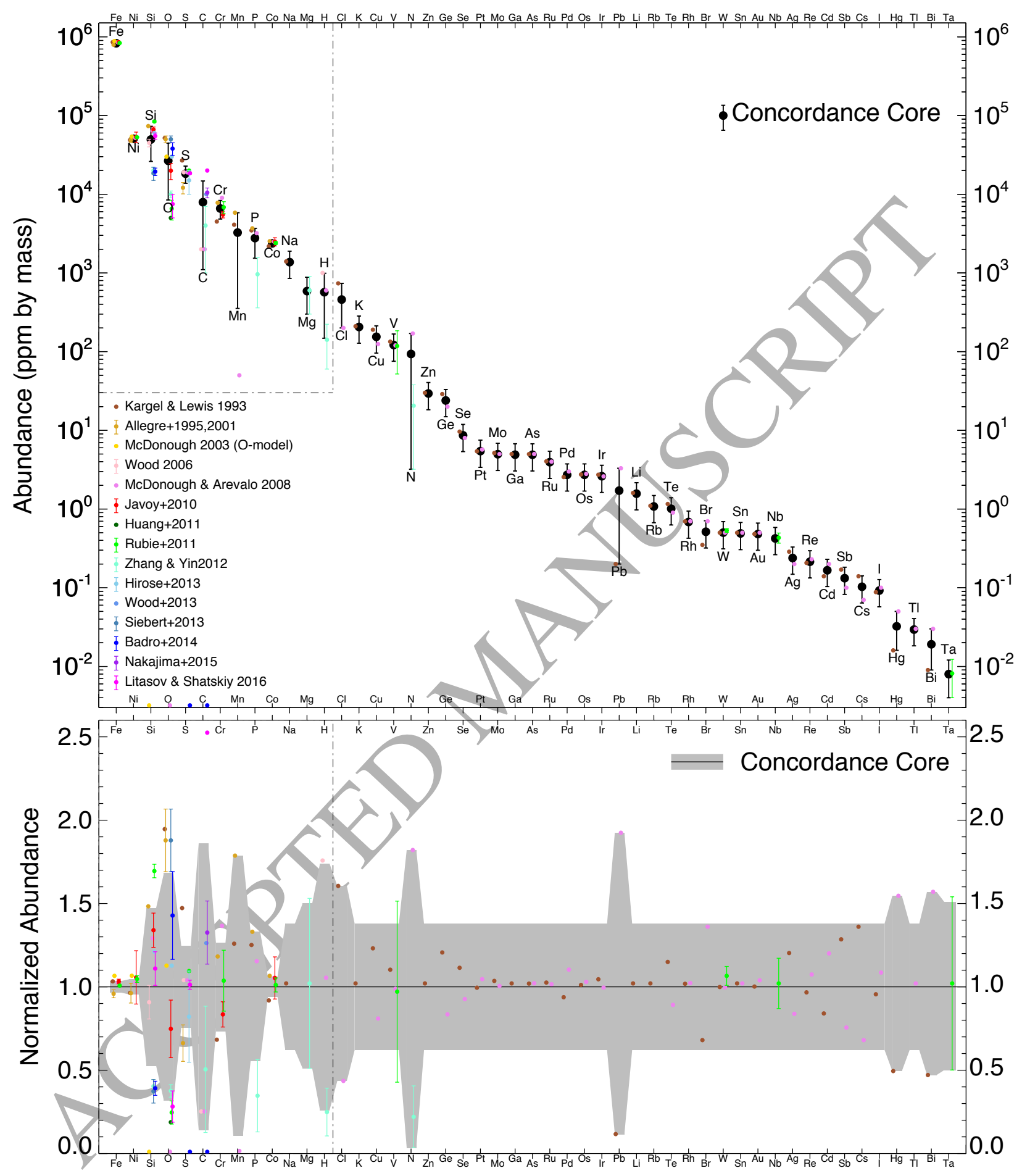

Decreasing Core Abundance $\rightarrow$

Figure 3: Recent literature estimates of 49 elemental abundances in the Earth's core and our concordance estimates constructed from them. Elements are plotted in order of decreasing core abundance. The upper panel plots ppm by mass. In the lower panel, literature values have been normalized to our core concordance estimates. The grey band in the lower panel indicates our estimate of the uncertainties on the concordance values. $79 \%$ of the literature points fall within this band. The dashed boxes on the left in both panels contain the 13 most abundant elements and are zoomed-in on in Fig. 4. Our core concordance ppm estimates have been rescaled down by $2.0 \%$ to constrain their sum to equal $10^{6}$. We have not rescaled the literature values (see Appendix C, Table C.4). 


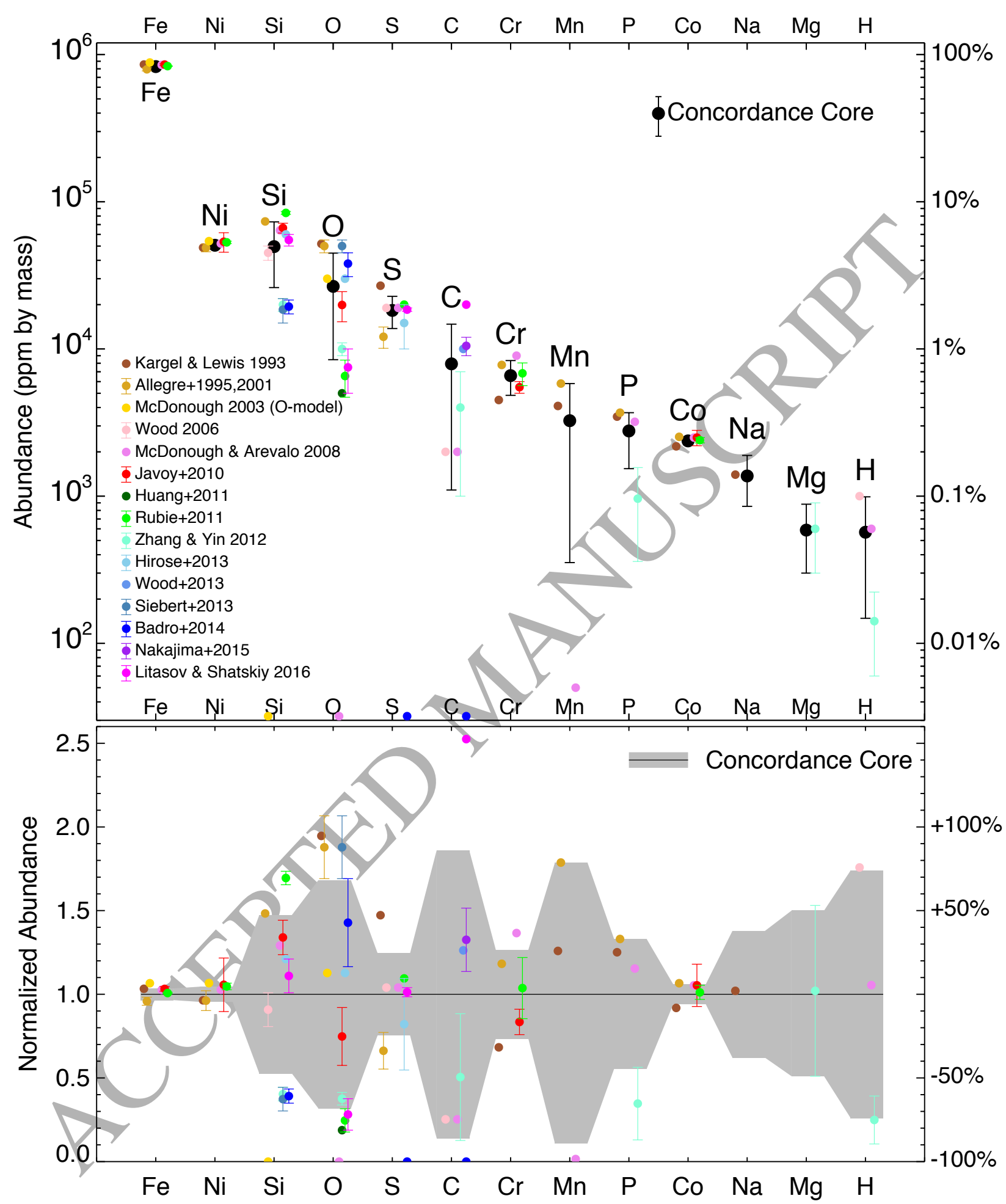

Figure 4: Zoom-in of the 13 most abundant elements contained in the dashed boxes in both panels of the previous figure. The sum of the Fe and $\mathrm{Ni}$ abundances make up $87.90 \pm 2.92 \%$ of the total core mass. The 5 most abundant elements (Fe, Ni, Si, O, S) make up $97.34_{-4.18}^{+2.66} \%$ while the 13 most abundant elements plotted here make up $99.89_{-4.11}^{+0.11} \%$ of the total core mass. See Table 1 for details. The uncertainties on Si and O are not independent since to account for the density deficit in the core, high values of Si probably coincide with low values of $\mathrm{O}$, and vice versa. 
Table 3: Core mass fraction in the Earth

\begin{tabular}{|c|c|c|}
\hline Reference & Core mass fraction (wt $\%$ ) & Comments \\
\hline Birch (1964) & 32.4 and 32.7 & solutions from two sets of density-velocity relations \\
\hline Anderson and Kovach (1967) & 32.5 & $\begin{array}{l}\text { see also Anderson (1989), using density profiles from Dziewonski and Anderson (1981) } \\
\text { Preliminary Reference Earth Model (PREM model) based on seismological constraints. }\end{array}$ \\
\hline Yoder (1995) & 32.3 & $\begin{array}{l}\text { derived from estimates of the masses of inner core, outer core, and the Earth: } 96.75 \times 10^{21} \\
\mathrm{~kg}, 1835 \times 10^{21} \mathrm{~kg} \text {, and } 5973.6 \times 10^{21} \mathrm{~kg} \text { respectively; The GEM-T2 Gravitation Model } \\
\text { (Marsh et al., 1990) and the PREM model (Dziewonski and Anderson, 1981) are cited. }\end{array}$ \\
\hline Allègre et al. $(1995,2001)$ & 32.5 & $\begin{array}{l}\text { derived from assumed masses of primitive mantle and core: } 4090 \times 10^{21} \mathrm{~kg} \text { and } 1967 \times 10^{21} \\
\mathrm{~kg} \text {, respectively. Thus a total Earth mass of } 6057 \times 10^{21} \text { is assumed; Anders (1977), Morgan } \\
\text { and Anders (1980) and Wanke and Dreibus (1988) are cited. }\end{array}$ \\
\hline McDonough (2003) & 32.3 & cites Yoder (1995) \\
\hline Javoy et al. (2010) & 32.4 & no citations given \\
\hline Hirose et al. (2013) & 33 & no citations given \\
\hline Zeng (2015) & 32.5 & no citations given \\
\hline This work & $32.5 \pm 0.3$ & $\begin{array}{l}\text { seismological constraints based on the Dziewonski and Anderson (1981) PREM model, the } \\
\text { Kennett et al. (1995) ak135 model, and the radii of inner core and outer core: } 3480 \pm 1 \mathrm{~km} \\
\text { and } 1218 \pm 3 \mathrm{~km} \text {, respectively, from Souriau and Calvet (2015), along with the constraint of } \\
\text { total mass of the Earth }\left(5972.2 \pm 0.6 \times 10^{21} \mathrm{~kg} \text {, the Astronomical Almanac Online, USNO- }\right. \\
\text { UKHO.). See details in Section } 4.1 \text {. }\end{array}$ \\
\hline
\end{tabular}

\section{Composition of the Bulk Earth}

\subsection{The Core Mass Fraction}

The elemental abundances of bulk Earth are the weighted average of the elemental abundances of primitive mantle and core. The accuracy of bulk Earth abundances therefore depends on the accuracy of the core mass fraction. However, literature values of the core mass fraction (Table 3 ) vary, and none has a reported uncertainty.

Our calculation of the core mass fraction is based on the two standard Earth radial density profile models: PREM (Dziewonski and Anderson, 1981) and ak135 (Kennett et al., 1995). We take 3480 $\pm 1 \mathrm{~km}$ for the core-mantle boundary $(\mathrm{CMB})$ and $1218 \pm 3 \mathrm{~km}$ for the inner core boundary (ICB) (Souriau and Calvet, 2015). We take $5972.2 \pm 0.6 \times 10^{21} \mathrm{~kg}$ as the total mass of the Earth ${ }^{1}$. This recent update of the Earth's mass is due to more precise (and accurate) estimates of Newton's constant. We renormalize the radial density profiles of core and mantle to this new lower Earth mass, which results in an overall density lower by $0.00158 \mathrm{~g} / \mathrm{cm}^{3}$. We assume a typical uncertainty of $1 \%$ in the density profile models (Brian Kennett, personal communication). We include the correlation between core and mantle densities, the uncertainties of the ICB and the CMB, and the fact that an increase of radius can be compensated by a lower density. Thus, we obtain estimates for the mass fraction of the inner core $\left(f_{i c}\right): f_{i c}=1.630 \pm 0.004 \mathrm{wt} \%$ and of the outer core $\left(f_{o c}\right): f_{o c}=30.840 \pm 0.296 \mathrm{wt} \%$. The mass fraction of the total core is $f_{i c}+f_{o c}=f_{\text {core }}=32.5 \pm 0.3 \mathrm{wt} \%$. Correspondingly, the weighting factors for estimating the bulk Earth composition are $f_{\text {core }}$ and $1-f_{\text {core }}$ for the core and PM respectively (Eq. A.5).

\subsection{Concordance Bulk Earth Estimate}

Based on our concordance estimates of the elemental abundances of the primitive mantle and core, we estimate the bulk elemental abundances of the Earth with their weighted sum ( Eq. A.5 ). The resultant bulk Earth composition and its associated uncertainty ( Eq. A.6) are listed in column 7 of Table 1. The construction of bulk Earth abundances from primitive mantle and core for each element is plotted in Figs. 5a and 5b to demonstrate the geochemical differentiation between PM and core. In Fig. 5b we normalize the elemental abundances of PM and core to the concordance bulk Earth abundance and plot them as a function of $50 \%$ condensation temperature (Lodders, 2003).

\footnotetext{
1 “2016 Selected Astronomical Constants” in The Astronomical Almanac Online, USNO-UKHO.
} 


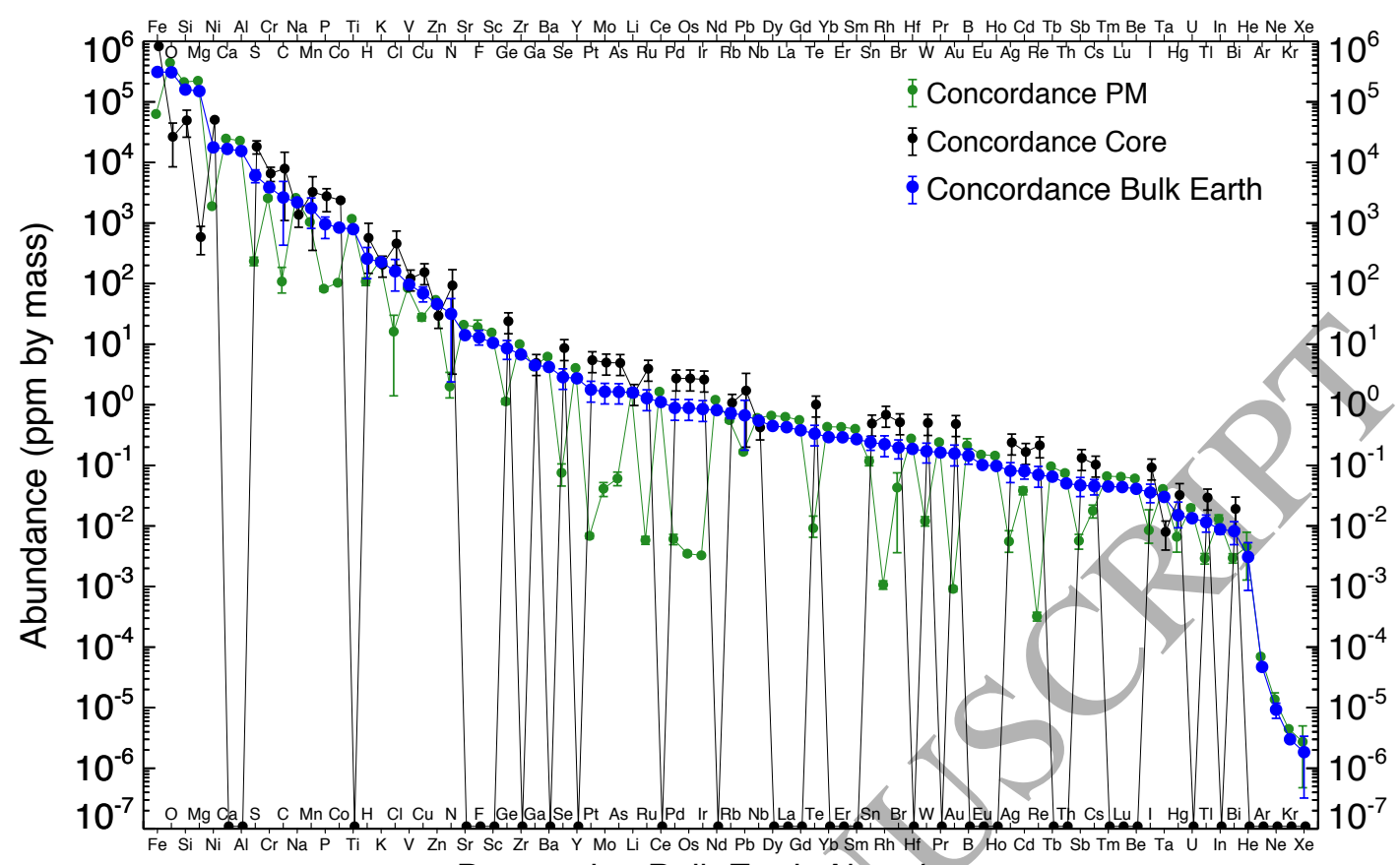

Decreasing Bulk Earth Abundance $\rightarrow$

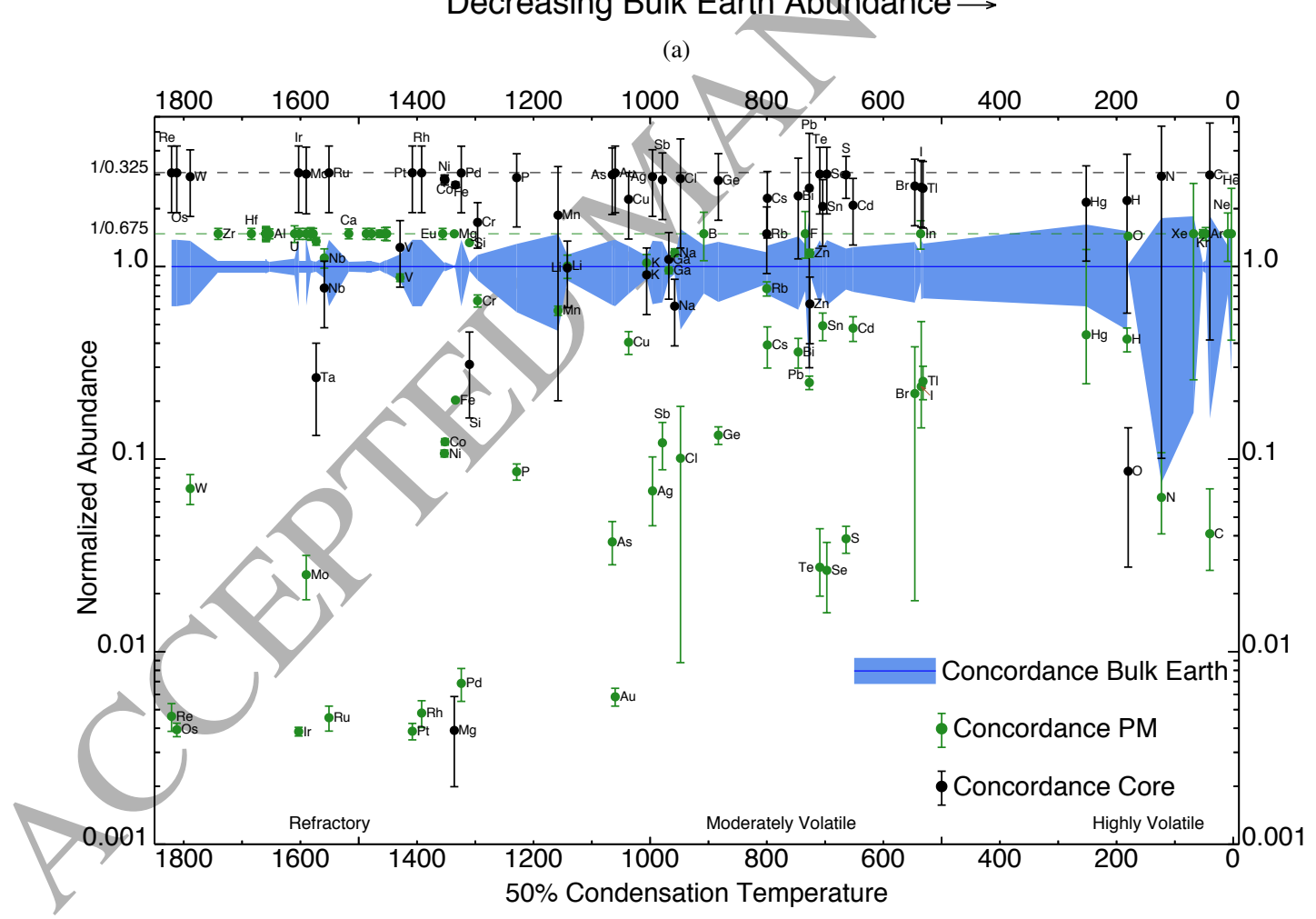

(b)

Figure 5: (a) The weighted sums of the concordance PM and the concordance core produce our concordance estimates of elemental abundances of the bulk Earth. The core abundances are weighted by our estimate of the core mass fraction: $32.5 \pm 0.3$ wt $\%$. The PM abundances are weighted by $67.5 \pm 0.3 \mathrm{wt} \%(=100-32.5)$. Elements with nil or extremely low abundance in the core are plotted on the $\mathrm{x}$-axis. (b) Concordance PM and core abundances normalized to the concordance bulk Earth abundances and plotted as a function of 50\% condensation temperatures (Lodders, 2003). Refractory elements are on the left, volatiles on the right. Refractory lithophile elements are the green points at normalized abundance of $\sim 1.5(\approx 1 / 0.675)$. Refractory siderophiles are the black points at a normalized abundance of $\sim 3(\approx 1 / 0.325)$. 
Our main results are shown in Fig. 6 which shows the comparison of our concordance estimate of bulk Earth composition and the recent bulk Earth compositional datasets of Allègre et al. (2001), McDonough (2003), and McDonough and Arevalo (2008). The light blue band in the lower panel indicates our estimate of the uncertainties on the concordance values. $70 \%$ of the literature points fall within this band. Fig. 7 zooms in on the comparison of the 15 most abundant elements, which account for $99.90_{-1.49}^{+0.10} \mathrm{wt} \%$ of the bulk Earth composition.

\section{Discussion}

\subsection{Comparison with Previous Estimates}

Compared to our concordance values, the abundances of some elements reported by McDonough (2003) and McDonough and Arevalo (2008) are significantly different (where the significance of the difference can only be based on our reported uncertainties). Relative to our values, their abundances for $\mathrm{Mg}, \mathrm{Cr}, \mathrm{Br}, \mathrm{Sn}, \mathrm{Cd}, \mathrm{B}$ and $\mathrm{Be}$ are significantly higher, while their abundances for $\mathrm{O}, \mathrm{Na}, \mathrm{Mn}, \mathrm{Zn}, \mathrm{Ga}, \mathrm{Li}, \mathrm{Rb}, \mathrm{Nb}, \mathrm{Ta}$ and $\mathrm{In}$ are significantly lower, where "significant" means their value is outside our estimate of the uncertainty (Figs, 6 \& 7).

The bulk elemental abundances with uncertainties in Allègre et al. (2001) were determined systematically by a carbonaceous chondrite correlation line, in combination with the abundances of siderophile and chalcophile elements in Allègre et al. (1995). These works largely depend on the accuracy of the analyses of CI, CM, CO and CV meteorites compiled in Wasson (1988). For many elements, their mean values and/or their uncertainties are different from our results. For example, relative to our values, the Allègre et al. (2001) abundances for $\mathrm{O}, \mathrm{Si}, \mathrm{Mg}, \mathrm{Sn}, \mathrm{Rh}, \mathrm{B}$ and $\mathrm{Cd}$ are high, while their abundances for $\mathrm{Fe}, \mathrm{K}, \mathrm{Cl}, \mathrm{Zn}, \mathrm{N}, \mathrm{F}, \mathrm{Ga}, \mathrm{Tl}, \mathrm{He}$ and $\mathrm{Ar}$ are low. One reason for these differences are the different assumptions made. For example, the estimate of nitrogen abundance in the Earth in Allègre et al. (2001) is based on the atmospheric inventories while our estimate is based on the comparison of Earth's mantle with carbonaceous chondrite data in McDonough and Arevalo (2008) and Palme and O'Neill (2014). This latter assumption is more widely accepted and better constrained. Allègre et al. (2001) deduced Earth's chlorine abundance from the $\mathrm{Cl} / \mathrm{Ba}$ ratio in MORB, while our PM sources (Lyubetskaya and Korenaga, 2007; McDonough and Arevalo, 2008) derived $\mathrm{Cl}$ from the $\mathrm{Cl} / \mathrm{K}$ ratio in MORB (Sect. 2.2). Other reasons for the abundance differences and differences in uncertainties are updates to PM and core abundances, and the use of slightly different core mass fractions: McDonough (2003) or McDonough and Arevalo (2008) (32.3 wt\%), Allègre et al. (2001) (32.5 wt\%), and this work $(32.5 \pm 0.3 \mathrm{wt} \%)$.

Based on Eq. B.1, we quantify the significance of the deviation of our concordance bulk Earth abundances from previous estimates (Allègre et al., 2001; McDonough, 2003; McDonough and Arevalo, 2008). Among our new bulk elemental abundances, 6 elements ( $\mathrm{Mg}, \mathrm{Sn}, \mathrm{Br}, \mathrm{B}, \mathrm{Cd}$ and $\mathrm{Be}$ ) are more than $\sim 1 \sigma$ below previous estimates, and 14 elements (Na, K, Cl, Zn, Sr, F, Ga, Rb, Nb, Gd, Ta, He, Ar, and Kr) are more than $\sim 1 \sigma$ above previous estimates (see Table B.4).

The reasons for these significant abundance differences include different assumptions and our inclusion of updated $\mathrm{PM}$ and core abundances. $\mathrm{Mg}$ and $\mathrm{Na}$ are the most abundant elements for which our new estimates deviate by more than $\sim 1.5 \sigma$ from previous estimates. Our Mg PM abundance of $22.3 \pm 0.2 \mathrm{wt} \%$ is $\sim 2.5 \sigma$ lower than the $22.8 \mathrm{wt} \%$ of McDonough and Arevalo (2008) and 5 $\sigma$ lower than the $23.4 \mathrm{wt} \%$ of Allègre et al. (2001) (deduced from their $15.8 \mathrm{wt} \% \mathrm{Mg}$ bulk abundance and a $67.5 \% \mathrm{PM}$ mass fraction). Our lower bulk Earth Mg abundance predominantly comes from the lower PM Mg abundance in Palme and O'Neill (2014). Our bulk Earth Na abundance is higher than McDonough and Arevalo (2008) and Allègre et al. (2001) because unlike those authors, we have included Na in the core:1372 \pm 519 ppm (Kargel and Lewis, 1993).

Palme and O'Neill (2014) state that the 6 most abundant elements in the PM make up $98.41 \pm 0.01 \mathrm{wt} \%$. We get a significantly higher value with a much larger uncertainty: $98.96 \pm 0.72 \mathrm{wt} \%$. McDonough (2016) states (based on chondritic models) that $\mathrm{Fe}, \mathrm{O}, \mathrm{Si}$ and $\mathrm{Mg}$ make up more than $90 \%$ of the mass for the bulk Earth and the addition of $\mathrm{Ni}, \mathrm{Ca}, \mathrm{Al}$ and $\mathrm{S}$ accounts for more than $98 \%$ by mass. Consistent with these estimates, we find $92.99 \pm 1.45 \mathrm{wt} \%$ for $\mathrm{Fe}, \mathrm{O}, \mathrm{Si}$ and $\mathrm{Mg}$ and $98.59_{-1.47}^{+1.41} \mathrm{wt} \%$ with the addition of $\mathrm{Ni}, \mathrm{Ca}, \mathrm{Al}$ and $\mathrm{S}$. 

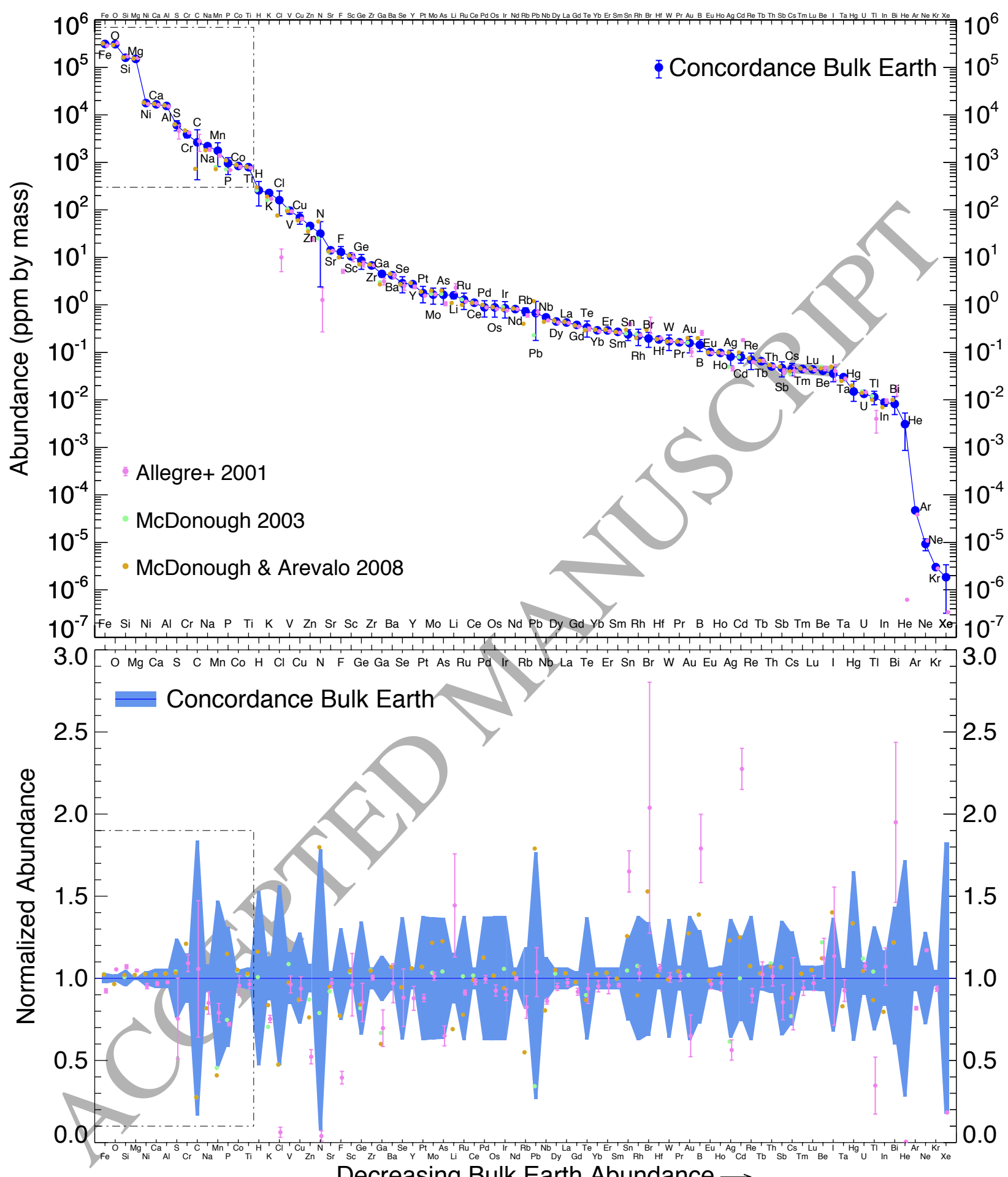

Figure 6: Comparison of our concordance bulk Earth elemental abundances (last column of Table 1) with the recent estimates of Allègre et al. (2001), McDonough (2003), and McDonough and Arevalo (2008). Elements for which McDonough (2003) and McDonough and Arevalo (2008) reported identical values are plotted as McDonough and Arevalo (2008) points. In the lower panel the literature values from the top panel are normalized to our concordance values. The blue band in the lower panel indicates our estimate of the uncertainties on the concordance values. $70 \%$ of the literature points fall within this band. The sum of our bulk Earth abundances is $10^{6}$ since we have rescaled the concordance PM and core abundances to $10^{6}$ (see Appendix C). The literature abundances have not been rescaled to ensure their abundances sum to $10^{6}$. The dashed boxes on the left in both panels contain the 15 most abundant elements and are zoomed-in on in Fig. 7. 


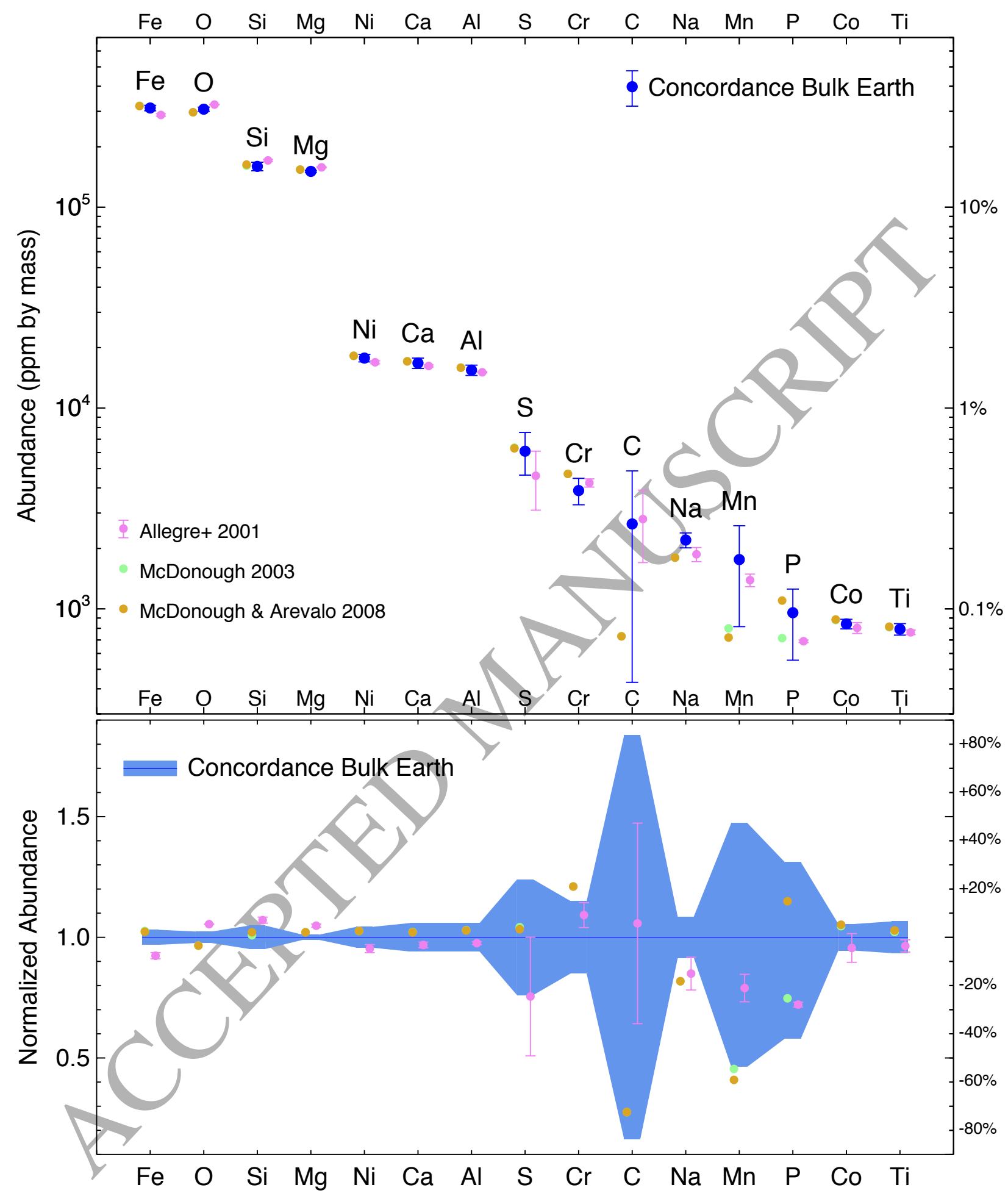

Figure 7: Zoom-in of the 15 most abundant elements contained in the dashed boxes in both panels of the previous figure. The sum of the abundances of the 4 most abundant elements (Fe, O, Si, Mg) make up $92.99 \pm 1.45 \%$ of the total mass of the Earth. The 8 most abundant elements (Fe, O, Si, $\mathrm{Mg}, \mathrm{Ni}, \mathrm{Ca}, \mathrm{Al}, \mathrm{S})$ make up $98.59_{-1.47}^{+1.41} \%$, while the 15 most abundant elements plotted here make up $99.90_{-1.49}^{+0.10} \%$ of the total mass of the Earth. Our $\mathrm{Mg}$ abundance is significantly lower than previous estimates. Our estimates of the uncertainties allow the evaluation of the significance of such differences. Reported values without uncertainties do not allow such a comparison. 
Fig. 8 presents another way to present our PM and bulk Earth compositions (with uncertainties) in comparison with the latest compilation (Palme et al., 2014) of CI chondritic abundances. In Fig. 8, we have normalized both the bulk Earth and PM abundances to the highly refractory element $\mathrm{Al}$ and $\mathrm{CI}$ chondrites. More specifically, for a generic elemental abundance $X$, for the bulk Earth we plot: $(X / \mathrm{Al})_{\text {Earth }} /(X / \mathrm{Al})_{\mathrm{CI}}$ and for the concordance PM we plot: $(X / \mathrm{Al})_{\mathrm{PM}} /(X / \mathrm{Al})_{\mathrm{CI}}$. This figure can be directly compared with Fig. 1 of Wood et al. (2006), Fig. 1 of Carlson et al. (2014) and Fig. 21 of Palme and O'Neill (2014). Those three figures have been normalized to Mg and CI chondrites. Because of their large abundances, $\mathrm{Mg}$ or $\mathrm{Si}$ have often been chosen as a normalization reference element (McDonough, 2003; Palme and O’Neill, 2014; Carlson et al., 2014; Litasov and Shatskiy, 2016). However both Mg and $\mathrm{Si}$ are not strictly refractory elements. They both have condensation temperatures slightly lower than the transition or critical temperature ( $1400 \mathrm{~K})$, below which the devolatilization of the Earth, compared to CI, is clear. Thus, with an $\mathrm{Mg}$ normalization, a slight depletion of bulk Earth $\mathrm{Mg}$ compared to $\mathrm{CI}$ chondritic $\mathrm{Mg}$, is misrepresented as an enrichment of refractory lithophiles.

Our PM analysis does not include estimates from Javoy et al. (2010) who only report the PM abundances of ten elements $(\mathrm{O}, \mathrm{Mg}, \mathrm{Si}, \mathrm{Fe}, \mathrm{Al}, \mathrm{Ca}, \mathrm{Ti}, \mathrm{Ni}, \mathrm{Cr}$, and $\mathrm{Co})$ based on their enstatite chondrite model. If these abundances were included in our analysis they would lower $\mathrm{Al}, \mathrm{Ca}$ and $\mathrm{Ti}$ without lowering the other RLEs such as $\mathrm{Be}, \mathrm{Sc}, \mathrm{Sr}$, $\mathrm{Nb}, \mathrm{REE}$ (Rare Earth Elements), Th and U. Also, enstatite chondrites are silica-enriched (but oxygen depleted?), from which it is problematic to construct the silica-poor (peridotitic) terrestrial model (Fitoussi and Bourdon, 2012; Jellinek and Jackson, 2015).

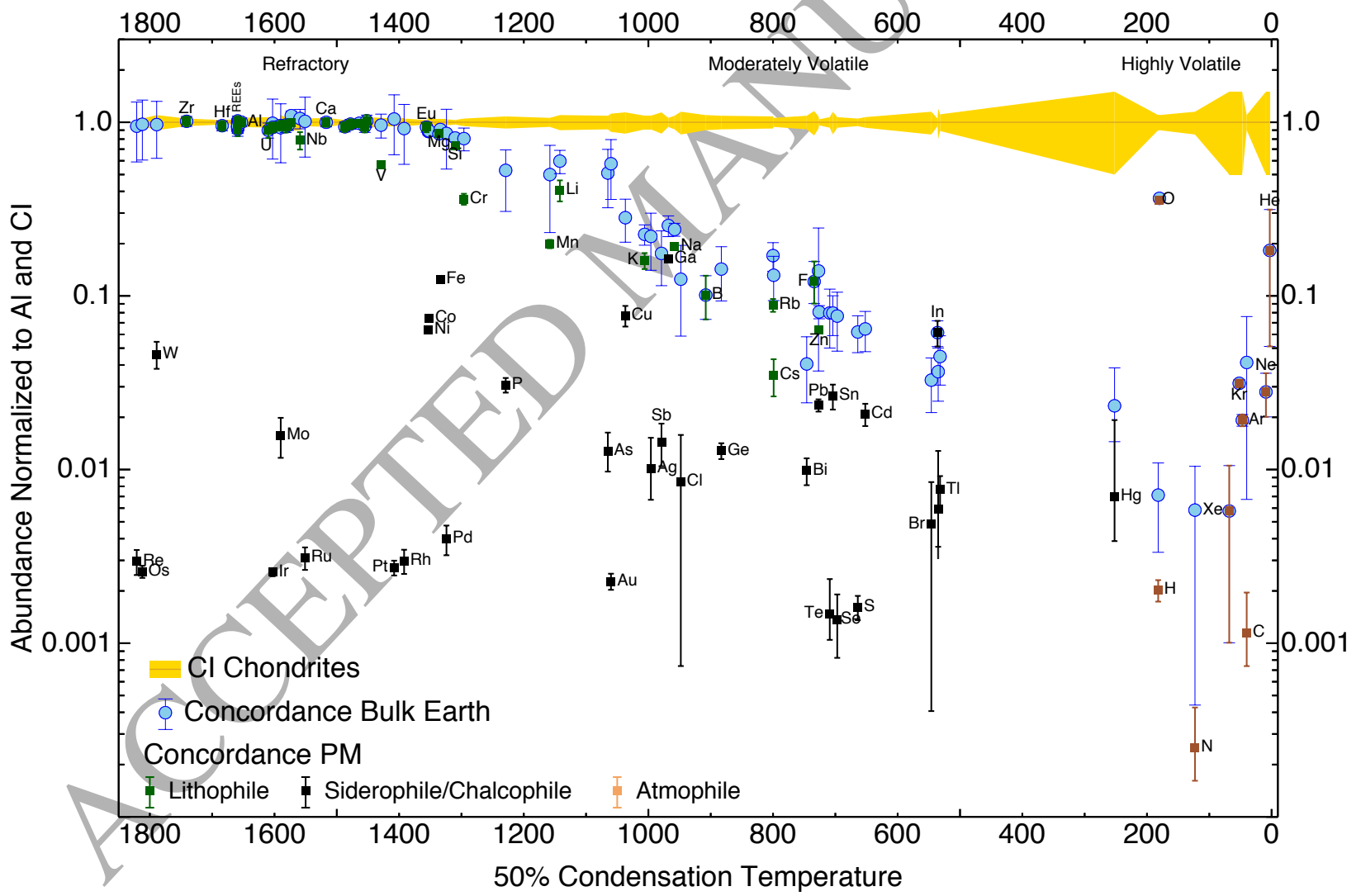

Figure 8: Our concordance bulk Earth and primitive mantle abundances normalized to $\mathrm{Al}$ and $\mathrm{CI}$ chondrites. Thus, for a generic element $X$, the bulk Earth (blue dots) we plot: $(X / \mathrm{Al})_{E a r t h} /(X / \mathrm{Al})_{C I}$ and analogously for the concordance PM (square points) we plot: $(X / \mathrm{Al})_{P M} /(X / \mathrm{Al})_{C I}$. Both are plotted as a function of $50 \%$ condensation temperatures (Lodders, 2003). CI abundances and uncertainties are from Table 3 of Palme et al. (2014), except for the uncertainties of the noble gases which we have set at the same $\pm 50 \%$ uncertainty of $\mathrm{Hg}$. 


\subsection{Unresolved Issues}

We have assumed that the bulk Earth consists of the primitive mantle and the core, and that the primitive mantle is a reservoir with the composition of the present-day Earth's mantle, crust and surface inventories taken together. Contributions of late accretion to the formation of a secondary atmosphere of the Earth introduces some ambiguity to this definition. We also neglected Earth's primary atmosphere (likely dominated by H and He) formed at the stage of solar nebula. In limiting our calculations to the prevalent standard models of the PM (Lyubetskaya and Korenaga, 2007; McDonough and Arevalo, 2008; Palme and O'Neill, 2014) we ignore the possible heterogeneity between the lower mantle and the upper mantle. Impact erosion is another issue that could have changed the PM abundances of incompatible lithophile elements. If we considered the impact erosion model of O'Neill and Palme (2008), for example, the PM abundance of K would decrease by about a factor of 2 .

Recently, based on i) a high PM abundance of the chalcophile element In, ii) nucleosynthetic isotope anomalies and iii) high- and low-pressure-temperature metal-silicate partitioning data, Wang et al. (2016) suggested that Earth's moderately volatile element composition may not be chondritic.

Another concern could be that we compute weighted averages when combining the elemental abundances of the primitive mantle while we compute unweighted averages for the core. Considering the global consistency of the PM abundance datasets (i.e., Lyubetskaya and Korenaga, 2007; McDonough and Arevalo, 2008; Palme and O'Neill, 2014), computing weighted averages is appropriate. Unlike the PM, core compositional models are much more complex and mixed with experimental data. Furthermore, uncertainties are not given in the majority of reported core compositional estimates, so computing weighted averages is not an option.

The degree to which the bulk compositions of Venus and Mars are different from the Earth (Morgan and Anders, 1980; Wanke and Dreibus, 1988) or broadly similar (Taylor, 2013; Kaib and Cowan, 2015; Fitoussi et al., 2016) is still unclear.

\section{Summary and Conclusions}

As the solar nebula condensed, evaporated and fractionated to form the early Earth (Wood et al., 2006; Carlson et al., 2014), the chemical composition of the bulk Earth was set. From a heterogeneous set of literature values, we present the most complete lists of the elemental abundances with uncertainties of the primitive mantle (PM), the core and the bulk Earth (Table 1, Figs. 1-4,6,7). The four most abundant elements (O, Mg, Si, and Fe) make up $94.19 \pm 0.69 \%$ of the total PM mass. Fe-Ni alloy accounts for $87.90 \pm 2.92 \mathrm{wt} \%$ of the total mass of the core, and the major light elements in the core are $\mathrm{Si}, \mathrm{O}, \mathrm{S}, \mathrm{C}, \mathrm{Cr}, \mathrm{Mn}, \mathrm{P}, \mathrm{Co}, \mathrm{Na}, \mathrm{Mg}$ and $\mathrm{H}$ in order of decreasing abundance. The concordance bulk Earth abundances with uncertainties come from the weighted average of our concordance PM and core. The weighting factor for this average comes from our new estimate (with uncertainty) of the core mass fraction of the Earth: $32.5 \pm 0.3 \mathrm{wt} \%$. Our concordance estimate of bulk Earth composition is largely consistent with recent bulk elemental abundance estimates; $70 \%$ of the previous bulk elemental abundances are within the uncertainties of our concordance bulk elemental abundances. Compared to previous work, the most significant differences include: 1) our abundances of $\mathrm{Mg}, \mathrm{Sn}, \mathrm{Br}, \mathrm{B}, \mathrm{Cd}$ and $\mathrm{Be}$ are more than $\sim 1 \sigma$ lower, and 2) our abundances of $\mathrm{Na}, \mathrm{K}, \mathrm{Cl}, \mathrm{Zn}$, $\mathrm{Sr}, \mathrm{F}, \mathrm{Ga}, \mathrm{Rb}, \mathrm{Nb}, \mathrm{Gd}, \mathrm{Ta}, \mathrm{He}, \mathrm{Ar}$ and $\mathrm{Kr}$, more than $\sim 1 \sigma$ higher (Table B.4). This set of concordance estimates (with uncertainties) for the elemental abundances of PM, core and bulk Earth provides a reference that can be used to compare the Earth to the Sun, which will lead to a more precise devolatilization pattern, potentially applicable to exoplanets and their host stars.

\section{Acknowledgments}

We thank two anonymous reviewers for insightful comments. We acknowledge useful discussions with William F. McDonough, Jun Korenaga, Hugh O’Neill, Hrvoje Tkalăcić, Brian Kennett and Marc Norman. H.W. acknowledges support from a Prime Minister's Australia Asia Endeavour Award and an Australian National University Postgraduate Research Scholarship. 


\section{Appendix A. Concordance Estimates}

\section{Appendix A.1. Concordance PM Estimates}

For a given elemental abundance $X$, we compute the weighted mean $\bar{X}$ and variance $\sigma^{2}$ from:

$$
\begin{aligned}
\bar{X} & =\frac{\sum X_{i} / \sigma_{i}^{2}}{\sum 1 / \sigma_{i}^{2}} \\
\sigma^{2} & =\frac{1}{\sum 1 / \sigma_{i}^{2}}
\end{aligned}
$$

where the index $i$ refers to a data set and ranges from 1 to $N$. For most PM abundances $N=3$. Since Lyubetskaya and Korenaga (2007) did not report abundances for C, H, N and O, N = 2 for these elements. Lyubetskaya and Korenaga (2007) abundances for $\mathrm{Cl}$ and $\mathrm{Br}$ are inconsistent with $\mathrm{Cl}$ and $\mathrm{Br}$ from the other two data sets. Therefore, for these 2 elements, our concordance abundance is the unweighted mean (Eq. A.3) and we take as its uncertainty the range from the highest to the lowest reported abundance.

We have treated noble gases differently. Marty (2012) using an atmospheric model and Halliday (2013) using three different models (layered mantle, impact erosion, and basaltic glass) report the molar abundances of non-radiogenic nuclides of noble gases $\left({ }^{3} \mathrm{He},{ }^{20} \mathrm{Ne},{ }^{36} \mathrm{Ar},{ }^{84} \mathrm{Kr},{ }^{130} \mathrm{Xe}\right.$ ). We convert these to atomic abundances (by number) by dividing by their estimated terrestrial primordial isotopic fractions (Lodders et al. 2009). We then convert to mass ppm using the atomic weights from Wieser et al. (2013). Our concordance PM abundances for noble gases are the median value of the highest upper limit and the lowest lower limit among the four models. We use the upper and lower limits as the uncertainty.

\section{Appendix A.2. Concordance Core Estimates}

Since the literature core abundances are more scattered and model-dependent than the PM abundances, we compute our concordance core abundances as unweighted means and assign the standard deviations as uncertainties using:

$$
\begin{aligned}
\bar{X} & =\frac{1}{N} \sum_{i=1}^{N} X_{i} \\
\sigma^{2} & =\frac{\sum\left(X_{i}-\bar{X}\right)^{2}}{N-1}
\end{aligned}
$$

Little work has been done on trace elements in the core. Kargel and Lewis (1993) and McDonough and Arevalo (2008) provide estimates for many trace elements but their estimates are not independent. Thus Eq. A.4 severely under-estimates the uncertainty on the abundances of these trace elements. To compensate for this underestimate, we do the following. If the standard deviation from Eq. A.4 is smaller than $\pm 40 \%$ we assign an uncertainty of $40 \%$ (which is the average of the uncertainies of the 10 most abundant elements in the core). If the standard deviation computed from Eq. A.4 extends beyond the range of the reported values, we report the range of values as the uncertainty. For those elements with only one reported value (i.e. $\mathrm{N}=1$ ), Eq. A.4 is undefined. For these cases, we report as an uncertainty either $\pm 40 \%$, or the uncertainty on the single point, whichever is larger.

\section{Appendix A.3. Approach for concordance bulk Earth estimate}

The concordance elemental abundances of the bulk Earth for each element are computed by

$$
X=X_{\text {core }} f_{\text {core }}+X_{P M}\left(1-f_{\text {core }}\right)
$$

where, $f_{\text {core }}$ is the core mass fraction estimated in Sect. 4.1. The uncertainty $\sigma_{X}$, associated with the bulk elemental abundance, is calculated by the error propagation of the three uncertainties: $\sigma_{X_{P_{M}}}, \sigma_{X_{\text {core }}}$, and $\sigma_{f_{\text {core }}}$ of the PM elemental abundance, the core elemental abundance, and the core mass fraction, respectively:

$$
\sigma_{X}^{2}=\sigma_{X_{\text {core }}}^{2} f_{\text {core }}^{2}+\sigma_{X_{P M}}^{2}\left(1-f_{\text {core }}\right)^{2}+\sigma_{f_{\text {core }}}^{2}\left(X_{P M}-X_{\text {core }}\right)^{2}
$$




\begin{tabular}{|c|c|c|c|c|c|c|c|c|c|c|c|c|}
\hline 6 Eleı & ents w & h abun & ances $\mathrm{r}$ & ore the & $\sim 1 \sigma$ & elow p & evious & estimat & & & & \\
\hline $\mathrm{Mg}$ & $\mathrm{Cd}$ & $\mathrm{B}$ & $\mathrm{Be}$ & $\mathrm{Br}$ & $\mathrm{Sn}$ & & & & & & & \\
\hline-2.60 & -1.79 & -1.76 & -1.71 & -1.40 & -1.15 & & & & & & & \\
\hline $14 \mathrm{El}$ & nents & th abu & dances & nore th & $\mathbf{n} \sim 10$ & above & reviou & estim & & & & \\
\hline $\mathrm{Zn}$ & $\mathrm{Ga}$ & $\mathrm{Ar}$ & $\mathrm{Ta}$ & $\mathrm{Na}$ & $\mathrm{K}$ & $\mathrm{Rb}$ & $\mathrm{F}$ & $\mathrm{He}$ & $\mathrm{Cl}$ & $\mathrm{Nb}$ & $\mathrm{Kr}$ & $\mathrm{Sr}$ \\
\hline 3.04 & 2.36 & 2.34 & 1.82 & 1.75 & 1.75 & 1.64 & 1.63 & 1.39 & 1.38 & 1.30 & 1.20 & 1.19 \\
\hline
\end{tabular}

\section{Appendix B. Calculation of significance of deviation}

In Figs. 7 \& 8 we compare our concordance bulk estimates with three previous estimates of bulk Earth abundances. For some elements, all three previous estimates are higher, or lower. For these elements we compute the significance $S_{X}$, of this difference:

$$
S_{X}=\frac{1}{N} \sum_{i=1}^{N} \frac{X_{i}-X}{\sqrt{\sigma_{X_{i}}^{2}+\sigma_{X}^{2}}}
$$

where, $X$ is our concordance estimate of the elemental abundance and $\sigma_{X}^{2}$ is its variance from Eq. A.6. $X_{i}$ is the abundance reported in the $i$-th literature source, with $N$ being the total number of literature sources. Identical abundances reported in McDonough (2003) and in McDonough and Arevalo (2008) have only been used once in this calculation. $\sigma_{X_{i}}^{2}$ is its variance. If the source has no reported variance, we set $\sigma_{X_{i}}^{2}=0$.

Table B.4 lists the significances (more than $\sim 1 \sigma$ ) of the deviations between our concordance bulk Earth abundances and previous estimates (Allègre et al., 2001; McDonough, 2003; McDonough and Arevalo, 2008).

\section{Appendix C. Rescaling Data}

Elemental abundances are usually reported in ppm by mass. Thus, when all elements are estimated, the sum of their abundances should equal $10^{6}$. The abundances of all elements are usually not reported. We use this $\Sigma$ ppm $=10^{6}$ constraint to rescale our PM, core (and thus our bulk) abundances. Our concordance PM abundances summed to $0.997 \times 10^{6}$ so we rescaled all of our PM abundances up by $0.3 \%$. Our concordance core abundances summed to $1.020 \times$ $10^{6}$ so we rescaled all of our core abundances down by $2.0 \%$. In Table C.5 we sum the literature abundances (column 3 ). In column 4 we supplement the sums from column 3 with our rescaled concordance abundances. Deviations from $1.000 \times 10^{6}$ in column 5 indicate the level of inconsistency in the literature with the $\Sigma \mathrm{ppm}=10^{6}$ constraint, even after the missing elements have been supplemented with our concordance abundances. 


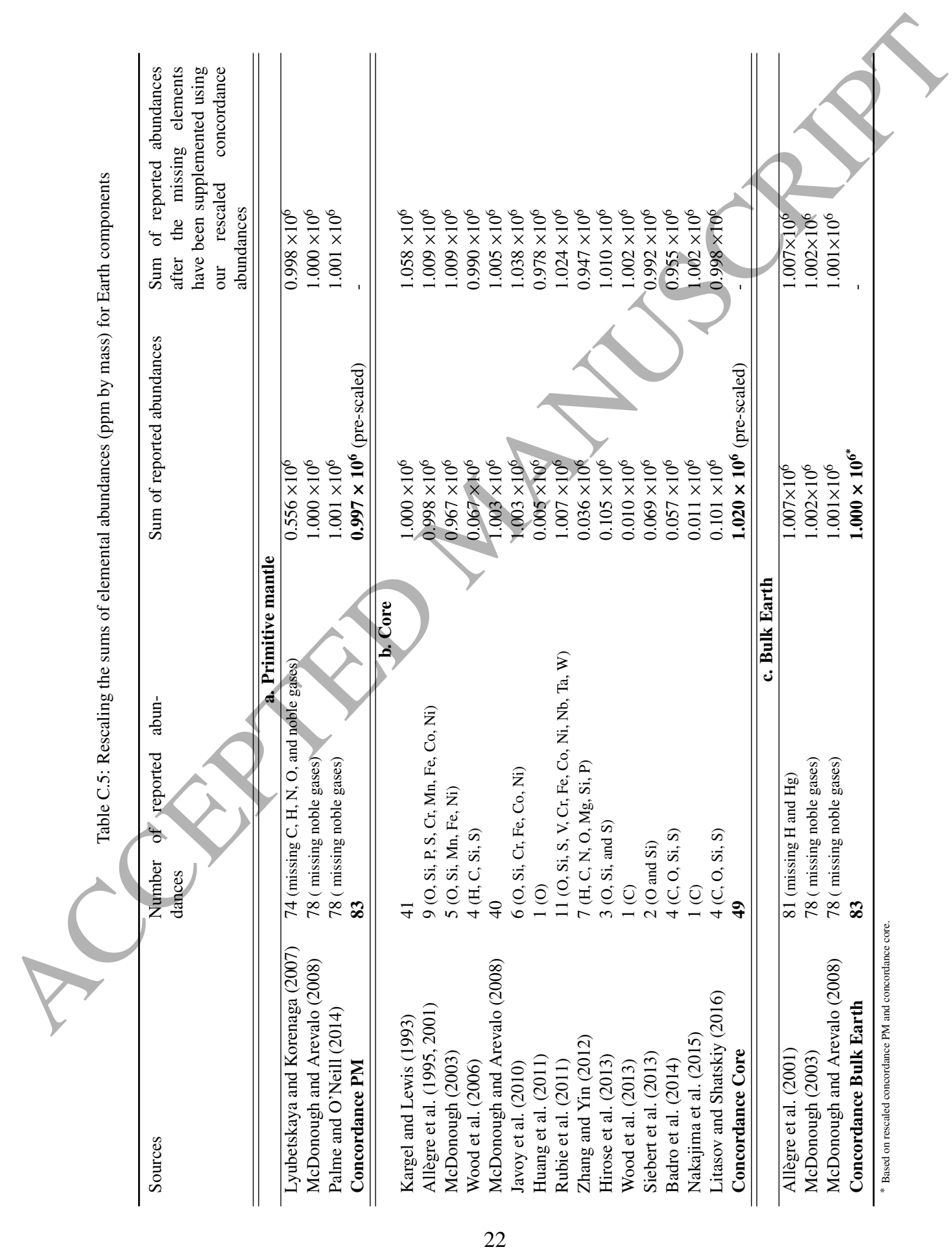




\section{References}

Allègre, C., Manhes, G., Lewin, E., 2001. Chemical composition of the Earth and the volatility control on planetary genetics. Earth. Planet. Sci. Lett. 185 (1-2), 49-69.

Allègre, C. J., Poirier, J. P., Humler, E., Hofmann, A. W., 1995. The chemical composition of the Earth. Earth Planet. Sci. Lett. 134 (3-4), 515-526.

Anders, E., 1977. Chemical compositions of the Moon, Earth , and eucrite parent body. Phil. Trans. R. So. Lond. A 285 (1327), 23-40.

Anderson, D. L., 1989. The crust and upper mantle. In: Theory of the Earth. No. 3. Blackwell Scientific Publications, pp. 45-62.

Anderson, D. L., Kovach, R. L., 1967. The composition of the terrestrial planets. Earth Planet. Sci. Lett. 3, 19-24.

Anderson, O., Isaak, D., 2002. Another look at the core density deficit of Earth's outer core. Phys. Earth Planet. Inter. 131 (1), 19-27.

Anderson, W., Ahrens, T., 1994. An equation of state for liquid iron and implications for the Earth's core. J. Geophys. Res. 99, 4273-4284.

Arevalo, R., McDonough, W. F., 2008. Tungsten geochemistry and implications for understanding the Earth's interior. Earth Planet. Sci. Lett. $272(3-4), 656-665$.

Arevalo, R., McDonough, W. F., Luong, M., 2009. The K/U ratio of the silicate Earth: Insights into mantle composition, structure and thermal evolution. Earth Planet. Sci. Lett. 278 (3-4), 361-369.

Badro, J., Côté, A. S., Brodholt, J. P., 2014. A seismologically consistent compositional model of Earth’s core. Proc. Natl. Acad. Sci. U. S. A., 2-5. Bellini, G., Benziger, J., Bonetti, S., Avanzini, M. B., Caccianiga, B., Cadonati, L., Calaprice, F., Carraro, C., Chavarria, A., Dalnoki-Veress, F., D’Angelo, D., Davini, S., de Kerret, H., Derbin, A., Etenko, A., Fiorentini, G., Fomenko, K., Franco, D., Galbiati, C., Gazzana, S., Ghiano, C., Giammarchi, M., Goeger-Neff, M., Goretti, A., Guardincerri, E., Hardy, S., Ianni, A., Ianni, A., Joyce, M., Kobychev, V. V., Koshio, Y., Korga, G., Kryn, D., Laubenstein, M., Leung, M., Lewke, T., Litvinovich, E., Loer, B., Lombardi, P., Ludhova, L., Machulin, I., Manecki, S., Maneschg, W., Manuzio, G., Meindl, Q., Meroni, E., Miramonti, L., Misiaszek, M., Montanari, D., Muratova, V., Oberauer, L., Obolensky, M., Ortica, F., Pallavicini, M., Papp, L., Perasso, L., Perasso, S., Pocar, A., Raghavan, R. S., Ranucci, G., Razeto, A., Re, A., Ricci, B., Risso, P., Romani, A., Rountree, D., Sabelnikov, A., Saldanha, R., Salvo, C., Schönert, S., Simgen, H., Skorokhvatov, M., Smirnov, O., Sotnikov, A., Sukhotin, S., Suvorov, Y., Tartaglia, R., Testera, G., Vignaud, D., Vogelaar, R. B., von Feilitzsch, F., Winter, J., Wojcik, M., Wright, A., Wurm, M., Xu, J., Zaimidoroga, O., Zavatarelli, S., Zuzel, G., 2010. Observation of geo-neutrinos. Phys. Lett. Sect. B Nucl. Elem. Part. High-Energy Phys. 687 (4-5), 299-304.

Bellini, G., Ianni, A., Ludhova, L., Mantovani, F., McDonough, W. F., 2013. Geo-neutrinos. Prog. Part. Nucl. Phys. 73, 1-34.

Birch, F., 1964. Density and composition of mantle and core. J. Geophys. Res. 69 (20), 4377.

Burbine, T. H., O'Brien, K. M., 2004. Determining the possible building blocks of the Earth and Mars. Meteorit. Planet. Sci. 39 (5), $667-681$.

Carlson, R. W., Garnero, E., Harrison, T. M., Li, J., Manga, M., McDonough, W. F., Mukhopadhyay, S., Romanowicz, B., Rubie, D., Williams, Q., Zhong, S., 2014. How Did Early Earth Become Our Modern World? Annu. Rev. Earth Planet. Sci. 42 (1), 151-178.

Chambers, J. E., 2001. Making more terrestrial planets. Icarus 152, 205.

Dauphas, N., Morbidelli, A., 2014. Geochemical and planetary dynamical views on the origin of Earth' atmosphere and oceans. In: Treatise Geochem. 6, 1-35.

Drake, M. J., Righter, K., 2002. Determining the composition of the Earth. Nature 416 (6876), 39-44.

Dziewonski, A. M., Anderson, D. L., 1981. Preliminary reference Earth model. Phys. Earth Planet. Inter. 25 (4), 297-356.

Fitoussi, C., Bourdon, B., 2012. Silicon Isotope Evidence Against an Enstatite Chondrite Earth. Science (80-. ). 335 (6075), 1477-1480.

Fitoussi, C., Bourdon, B., Wang, X., 2016. The building blocks of Earth and Mars: A close genetic link. Earth Planet. Sci. Lett. 434, 151-160.

Gando, A., Gando, Y., Hanakago, H., Ikeda, H., Inoue, K., Ishidoshiro, K., Ishikawa, H., Koga, M., Matsuda, R., Matsuda, S., Mitsui, T., Motoki, D., Nakamura, K., Obata, A., Oki, A., Oki, Y., Otani, M., Shimizu, I., Shirai, J., Suzuki, A., Takemoto, Y., Tamae, K., Ueshima, K., Watanabe, H., Xu, B. D., Yamada, S., Yamauchi, Y., Yoshida, H., Kozlov, A., Yoshida, S., Piepke, A., Banks, T. I., Fujikawa, B. K., Han, K., O’Donnell, T., Berger, B. E., Learned, J. G., Matsuno, S., Sakai, M., Efremenko, Y., Karwowski, H. J., Markoff, D. M., Tornow, W., Detwiler, J. A., Enomoto, S., Decowski, M. P., 2013. Reactor on-off antineutrino measurement with KamLAND. Phys. Rev. D - Part. Fields, Gravit. Cosmol. 88 (3), 1-10.

Gando, a., Gando, Y., Ichimura, K., Ikeda, H., Inoue, K., Kibe, Y., Kishimoto, Y., Koga, M., Minekawa, Y., Mitsui, T., Morikawa, T., Nagai, N., Nakajima, K., Nakamura, K., Narita, K., Shimizu, I., Shimizu, Y., Shirai, J., Suekane, F., Suzuki, a., Takahashi, H., Takahashi, N., Takemoto, Y., Tamae, K., Watanabe, H., Xu, B. D., Yabumoto, H., Yoshida, H., Yoshida, S., Enomoto, S., Kozlov, a., Murayama, H., Grant, C., Keefer, G., Piepke, a., Banks, T. I, Bloxham, T., Detwiler, J. a., Freedman, S. J., Fujikawa, B. K., Han, K., Kadel, R., O’Donnell, T., Steiner, H. M., Dwyer, D. a., McKeown, R. D., Zhang, C., Berger, B. E., Lane, C. E., Maricic, J., Miletic, T., Batygov, M., Learned, J. G., Matsuno, S., Sakai, M., Horton-Smith, G. a., Downum, K. E., Gratta, G., Tolich, K., Efremenko, Y., Perevozchikov, O., Karwowski, H. J., Markoff, D. M., Tornow, W., Heeger, K. M., Decowski, M. P., 2011. Partial radiogenic heat model for Earth revealed by geoneutrino measurements. Nat. Geosci. 4 (9), $647-651$

Halliday, A. N. 2013. The origins of volatiles in the terrestrial planets. Geochim. Cosmochim. Acta 105, 146-171.

Hemley, R. J., Mao, H.-K., 2001. In situ studies of iron under pressure: New windows on the Earth's core. Int. Geol. Rev. 43 (1), 1-30.

Hirose, K., Labrosse, S, Hernlund, J., 2013. Composition and State of the Core. Annu. Rev. Earth Planet. Sci. 41 (1), 657-691.

Huang, H., Fei, Y., Cai, L., Jing, F., Hu, X., Xie, H., Zhang, L., Gong, Z., 2011. Evidence for an oxygen-depleted liquid outer core of the Earth. Nature 479 (7374), 513-516.

Huang, Y., Chubakov, V., Mantovani, F., Rudnick, R. L., McDonough, W. F., 2013. A reference Earth model for the heat-producing elements and associated geoneutrino flux. Geochemistry, Geophys. Geosystems 14 (6), 2003-2029.

Javoy, M., Kaminski, E., Guyot, F., Andrault, D., Sanloup, C., Moreira, M., Labrosse, S., Jambon, A., Agrinier, P., Davaille, A., Jaupart, C., 2010. The chemical composition of the Earth: Enstatite chondrite models. Earth Planet. Sci. Lett. 293 (3-4), 259-268.

Jellinek, A. M., Jackson, M. G., 2015. Connections between the bulk composition, geodynamics and habitability of Earth. Nat. Geosci. 8 (8), 587-593.

Kaib, N. A., Cowan, N. B., 2015. The feeding zones of terrestrial planets and insights into Moon formation. Icarus 252 (Canup 2004), 161-174.

Kargel, J., Lewis, J., 1993. The composition and early evolution of Earth. Icarus 105 (1), 1-25.

Kennett, B. L. N., Engdahl, E. R., Buland, R., 1995. Constraints on seismic velocities in the Earth from traveltimes. Geophys. J. Int. 122 (1), $108-124$. 
Li, J., Fei, Y., 2014. Experimental constraints on core composition. In: Treatise on Geochemistry. Vol. 3. Elsevier, pp. $527-557$. Litasov, K. D., Shatskiy, A. F., 2016. Composition of the Earth's core: A review. Russ. Geol. Geophys. 57 (1), $22-46$.

Lodders, K., 2003. Solar System abundances and condensation temperatures of the elements. Astrophys. J. 591 (2), $1220-1247$.

Lodders, K., Palme, H., Gail, H.-P., 2009. Abundances of the elements in the Solar System. In: Trümper, J. (Ed.), Landolt- Börnstein, New Ser. Vol. VI/4B. Springer-Verlag, Ch. 4.4, pp. 560-598.

Lyubetskaya, T., Korenaga, J., 2007. Chemical composition of Earth's primitive mantle and its variance: 1. Method and results. J. Geophys. Res. Solid Earth 112 (3), 1-21.

Marsh, J. G., Lerch, F. J., Putney, B. H., Felsentreger, T. L., Sanchez, B. V., Klosko, S. M., Patel, G. B., Robbins, J. W., Williamson, R. G., Engelis, T. L., Eddy, W. F., Chandler, N. L., Chinn, D. S., Kapoor, S., Rachlin, K. E., Braatz, L. E., Pavlis, E. C., 1990. The GEM-T2 gravitational model. J. Geophys. Res. 95 (B13), 22043.

Marty, B., 2012. The origins and concentrations of water, carbon, nitrogen and noble gases on Earth. Earth Planet. Sci. Lett. 313-314 (1), 56-66.

Marty, B., Avice, G., Sano, Y., Altwegg, K., Balsiger, H., Hässig, M., Morbidelli, A., Mousis, O., Rubin, M., 2016. Origins of volatile elements (H, C, N, noble gases) on Earth and Mars in light of recent results from the ROSETTA cometary mission. Earth Planet. Sci. Lett. 441, $91-102$.

McDonough, W., 2003. Compositional model for the Earth's core. In: Carlson, R. W. (Ed.), Treatise on Geochemistry, 1st Edition. Vol. 2. Elsevier, pp. 547-568.

McDonough, W., Sun, S.-s., 1995. The composition of the Earth. Chem. Geol. 120 (3-4), 223-253.

McDonough, W. F., 2016. The Composition of the lower mantle and core. In: Terasaki, H., Fischer, R. A. (Eds.), Deep Earth Phys. Chem. Low. Mantle Core, Geophys. Monogr. Ser., 1st Edition. John Wiley \& Sons, Inc.

McDonough, W. F., Arevalo, R., 2008. Uncertainties in the composition of Earth, its core and silicate sphere. XXIII Conf. Neutrino Phys. Astrophys. J. Phys. Conf. Ser. 136 (2), 022006.

Mookherjee, M., Nakajima, Y., Steinle-Neumann, G., Glazyrin, K., Wu, X., Dubrovinsky, L., McCammon, C., Chumakov, A., 2011. High-pressure behavior of iron carbide (Fe7C3) at inner core conditions. J. Geophys. Res. Solid Earth 116, 1-13.

Morgan, J. W., Anders, E., 1980. Chemical composition of Earth, Venus, and Mercury. Proc. Natl. Acad. Sci. 77 (12), $6973-6977$.

Nakajima, M., Stevenson, D. J., 2015. Melting and mixing states of the Earth's mantle after the Moon-forming impact. Earth Planet. Sci. Lett. 427, $286-295$.

Nakajima, Y., Imada, S., Hirose, K., Komabayashi, T., Ozawa, H., Tateno, S., Tsutsui, S., Kuwayama, Y., Baron, A. Q. R., 2015. Carbon-depleted outer core revealed by sound velocity measurements of liquid iron-carbon alloy. Nat. Commun. 6 (May), 8942.

O'Neill, H., 1991. The origin of the moon and the early history of the earth-A chemical model. Part 2: The earth. Geochim. Cosmochim. Acta 55 (4), 1159-1172.

O’Neill, H. S. C., Palme, H., 1998. Composition of the silicate earth: implications for accretion and core formation. In: Jackson, I. (Ed.), Earth's Mantle Compos. Struct. Evol. Cambridge University Press, pp. 3-126.

O’Neill, H. S. C., Palme, H., 2008. Collisional erosion and the non-chondritic composition of the terrestrial planets. Philos. Trans. A. Math. Phys. Eng. Sci. 366 (1883), 4205-4238.

Palme, H., Lodders, K., Jones, A., 2014. Solar System abundances of the elements. In: Davis, A. M. (Ed.), Treatise Geochemistry Second Ed., 2nd Edition. Vol. 2. Elsevier Ltd., Ch. 2, pp. 15-36.

Palme, H., O’Neill, H., 2014. Cosmochemical estimates of mantle composition. In: Carlson, R. W. (Ed.), Treatise on Geochemistry, 2nd Edition. Vol. 3. Elsevier, pp. 1-39.

Palme, H., O’Neill, H. S. C., 2003. Cosmochemical estimatess of mantle composition. In: Carlson, R. W. (Ed.), Treatise on Geochemistry, 1st Edition. Vol. 2. Elsevier, pp. 1-38.

Poitrasson, F., Zambardi, T., 2015. An Earth-Moon silicon isotope model to track silicic magma origins. Geochim. Cosmochim. Acta 167, $301-312$.

Ricolleau, A., Fei, Y., Corgne, A., Siebert, J., Badro, J., 2011. Oxygen and silicon contents of Earth's core from high pressure metal-silicate partitioning experiments. Earth Planet. Sci. Lett. 310 (3-4), 409-421.

Ringwood, A., 1979. Origin of the Earth and Moon, 1st Edition. Springer-Verlag New York, Inc.

Rubie, D. C., Frost, D. J., Mann, U., Asahara, Y., Nimmo, F., Tsuno, K., Kegler, P., Holzheid, A., Palme, H., 2011. Heterogeneous accretion, composition and core-mantle differentiation of the Earth. Earth Planet. Sci. Lett. 301 (1-2), 31-42.

Saal, A. E., Hauri, E. H., Langmuir, C. H., Perfit, M. R., 2002. Vapour undersaturation in primitive mid-ocean-ridge basalt and the volatile content of Earth's upper mantle. Nature 419 (6906), 451-455.

Siebert, J., Badro, J., Antonangeli, D,, Ryerson, F. J., 2013. Terrestrial Accretion Under Oxidizing Conditions. Science (80-. ). 339 (6124), 11941197.

Souriau, A., Calvet, M., 2015. Deep Earth Structure: The Earth’s Cores. In: Treatise Geophys., 2nd Edition. Elsevier, Ch. 1.19, pp. 725-757.

Sramek, O., McDonough, W. F., Kite, E. S., Lekic, V., Dye, S. T., Zhong, S., 2013. Geophysical and geochemical constraints on geoneutrino fluxes from Earth's mantle. Earth Planet. Sci. Lett. 361, 356-366.

Stevenson, D. J., 1981. Models of the Earth's Core. Science (80-. ). 214 (4521), 611-619.

Sun, S. S., 1982. Chemical composition and origin of the earth's primitive mantle. Geochim. Cosmochim. Acta 46 (2), $179-192$.

Taylor, G. J., 2013. The bulk composition of Mars. Chemie der Erde - Geochemistry 73 (4), 401-420.

Vočadlo, L., 2007. Ab initio calculations of the elasticity of iron and iron alloys at inner core conditions: Evidence for a partially molten inner core? Earth Planet. Sci. Lett. 254 (1-2), 227-232.

Wang, Z., Laurenz, V., Petitgirard, S., Becker, H., 2016. Earth's moderately volatile element composition may not be chondritic: Evidence from In, Cd and Zn. Earth Planet. Sci. Lett. 435, 136-146.

Wanke, H., Dreibus, G., 1988. Chemical composition and accretion history of terrestrial planets. Phil. Trans. R. So. Lond. A $325,545-557$.

Wasson, B. Y. J. T., 1988. Compositions of chondrites. Philos. Trans. R. Soc. A Math. Phys. Eng. Sci. 325 (1587), $535-544$.

Wieser, M. E., Holden, N., Coplen, T. B., Böhlke, J. K., Berglund, M., Brand, W. A., De Bièvre, P., Gröning, M., Loss, R. D., Meija, J., Hirata, T., Prohaska, T., Schoenberg, R., O’Connor, G., Walczyk, T., Yoneda, S., Zhu, X.-K., 2013. Atomic weights of the elements 2011 (IUPAC Technical Report). Pure Appl. Chem. 85 (5), 1047-1078.

Wood, B. J., Li, J., Shahar, A., 2013. Carbon in the Core: Its Influence on the Properties of Core and Mantle. Rev. Mineral. Geochemistry 75 (1), 
$231-250$.

481 Wood, B. J., Walter, M. J., Wade, J., 2006. Accretion of the Earth and segregation of its core. Nature 441 (7095), 825-833.

482 Yoder, C., 1995. Global earth physics : a handbook of physical constants. In: Ahrens, T. (Ed.), AGU Ref. shelf. No. 1. pp. 1-31.

483 Zeng, L., 2015. Interior Structure and Chemistry of Solid Exoplanets. Doctoral dissertation, Harvard University.

484 Zhang, Y., Yin, Q.-Z., 2012. Carbon and other light element contents in the Earth's core based on first-principles molecular dynamics. Proc. Natl. Acad. Sci. 109 (48), 19579-19583.

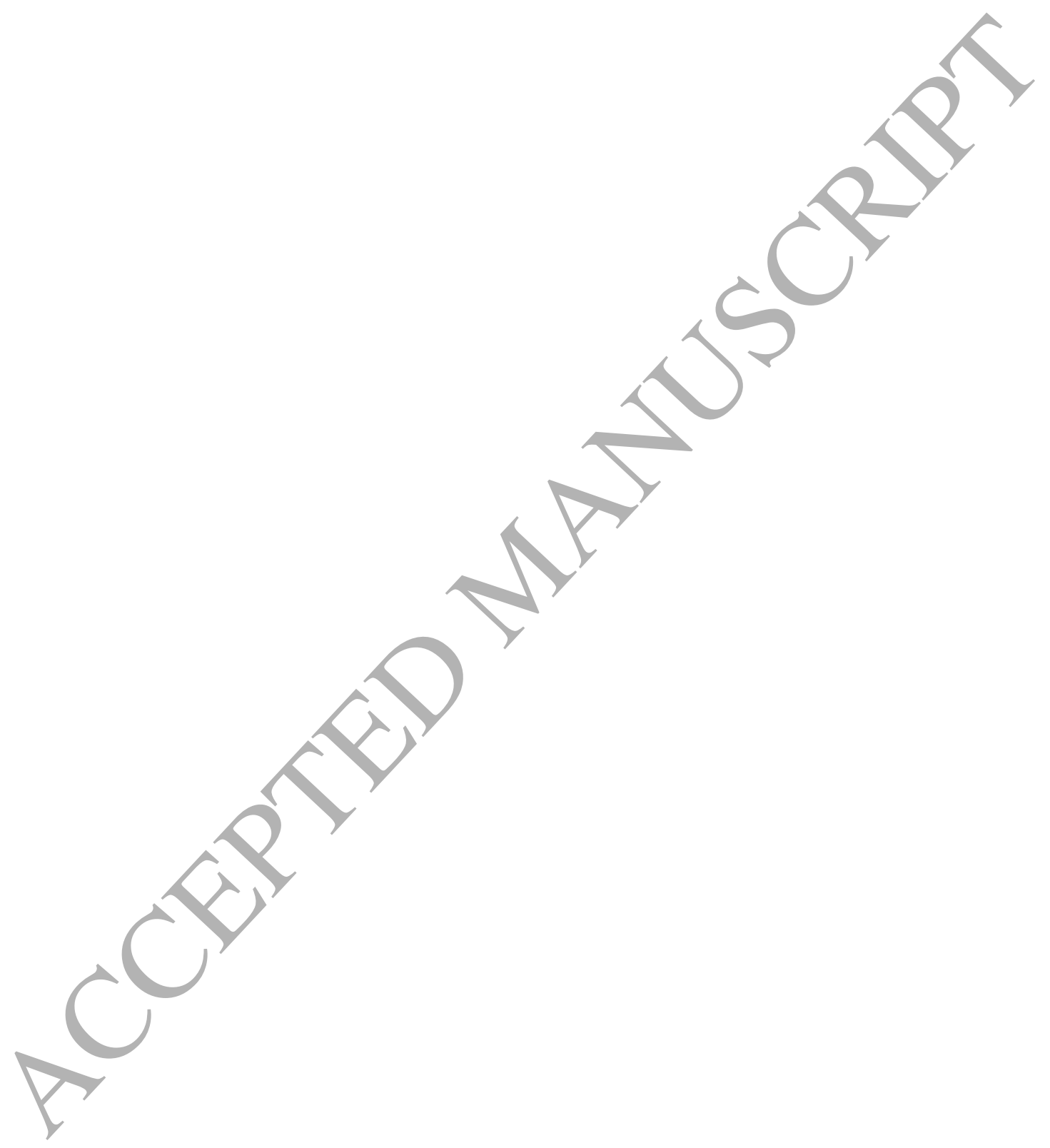

